\title{
A comprehensive observational filter for satellite infrared limb sounding of gravity waves
}

\author{
Q. T. Trinh ${ }^{1}$, S. Kalisch ${ }^{1}$, P. Preusse ${ }^{1}$, H.-Y. Chun ${ }^{2}$, S. D. Eckermann ${ }^{3}$, M. Ern ${ }^{1}$, and M. Riese ${ }^{1}$ \\ ${ }^{1}$ Institute of Energy and Climate Research, Stratosphere (IEK-7), Forschungszentrum Jülich, Jülich, Germany \\ ${ }^{2}$ Laboratory for Atmospheric Dynamics, Department of Atmospheric Sciences, Yonsei University, South Korea \\ ${ }^{3}$ Space Science Division, Naval Research Laboratory, Washington, D.C., USA \\ Correspondence to: Q. T. Trinh (t.trinh@fz-juelich.de)
}

Received: 2 October 2014 - Published in Atmos. Meas. Tech. Discuss.: 23 October 2014

Revised: 28 January 2015 - Accepted: 6 March 2015 - Published: 23 March 2015

\begin{abstract}
This paper describes a comprehensive observational filter for satellite infrared limb sounding of gravity waves. The filter considers instrument visibility and observation geometry with a high level of accuracy. It contains four main processes: visibility filter, projection of the wavelength on the tangent-point track, aliasing effect, and calculation of the observed vertical wavelength. The observation geometries of the SABER (Sounding of the Atmosphere using Broadband Emission Radiometry) and HIRDLS (High Resolution Dynamics Limb Sounder) are mimicked. Gravity waves (GWs) simulated by coupling a convective GW source (CGWS) scheme and the gravity wave regional or global ray tracer (GROGRAT) are used as an example for applying the observational filter. Simulated spectra in terms of horizontal and vertical wave numbers (wavelengths) of gravity wave momentum flux (GWMF) are analyzed under the influence of the filter. We find that the most important processes, which have significant influence on the spectrum are the visibility filter (for both SABER and HIRDLS observation geometries) and aliasing for SABER and projection on tangent-point track for HIRDLS. The vertical wavelength distribution is mainly affected by the retrieval as part of the "visibility filter" process. In addition, the short-horizontalscale spectrum may be projected for some cases into a longer horizontal wavelength interval which originally was not populated. The filter largely reduces GWMF values of very short horizontal wavelength waves. The implications for interpreting observed data are discussed.
\end{abstract}

\section{Introduction}

Gravity waves (GWs) play an important role in the dynamics of the middle atmosphere (e.g., McLandress, 1998; McIntyre, 1998; Kim et al., 2003; Alexander et al., 2010). Generated in the troposphere by various sources (e.g., orography, convection, spontaneous adjustment of jet streams), GWs propagate upwards with an increasing amplitude due to the exponential air density decline. This amplitude increase continues until the amplitude saturation level is reached, where GWs break, deposit momentum and accelerate or decelerate the atmosphere background flow. This process strongly depends on the refraction of the GWs by the background wind field, thus forming a two-way interaction between mean winds and GWs. Hence, GWs significantly affect the global circulation and are the main driver of the quasi-biennial oscillation (QBO) (e.g., Dunkerton, 1997; Ern and Preusse, 2009; Alexander and Ortland, 2010; Evan et al., 2012; Ern et al., 2014). In addition, gravity waves also play a key role in wind reversals in the mesosphere and lower thermosphere (Lindzen, 1981; Matsuno, 1982; Ern et al., 2013), and they cause the cold summer mesopause (e.g., Björn, 1984). Moreover, GWs are widely accepted as the main driver of the summertime branch of the stratospheric Brewer-Dobson circulation (Alexander and Rosenlof, 2003; Fritts and Alexander, 2003). Also, general circulation models predict an acceleration of Brewer-Dobson circulation in a warming climate, which is influenced by GWs (Garcia and Randel, 2008; Li et al., 2008; McLandress and Shepherd, 2009; Butchart et al., 2010). 
In most general circulation models (GCMs), in particular those for climate runs, the effects of GWs are treated via parameterizations since GWs are small-scale processes and are not resolved in these GCMs. These parameterizations, however, use some simplifying assumptions and have a number of free tunable parameters (Fritts and Alexander, 2003). Observations are therefore important to validate these parameterizations. Several studies used observations to constrain and to improve GW parameterizations (Ern et al., 2006; Preusse et al., 2009a; Orr et al., 2010; Choi et al., 2009, 2012; Geller et al., 2013). These studies, however, are limited in using only absolutes values of gravity wave momentum flux (GWMF), which have quite large uncertainties (Ern et al., 2004). In order to quantify these uncertainties and in order to capture the fact that a measured GWMF distribution, in general, may deviate from the true one in the atmosphere, the concept of the observational filter was introduced.

The importance of the observational filter was first pointed out by Alexander (1998). In her work for the MLS (microwave limb sounder), rocket sounding, and radiosonde measurements, the effects of the vertical resolution and of the analysis method were estimated, and the visibility was quantified as a function of the vertical wavelength. This function was applied to the spectrum given by a linear GW model. The resulting global maps agreed well with global maps from MLS observations (Wu and Waters, 1996b). Good agreement was also found with rocket sounding data (Eckermann et al., 1995) in terms of zonal mean GW variance. Moreover, modeled results of Alexander (1998) showed reasonable agreement with radiosonde measurements (Allen and Vincent, 1995) in terms of the seasonal cycle of GW energy density at midlatitudes.

Furthermore, significant differences in the morphology of GW-induced temperature variances between different limbsounding instruments result from different observational filters. This was first hypothesized by Alexander (1998) and tested by Preusse et al. (2000) for four satellite instruments: Cryogenic Infrared Spectrometers and Telescopes for the Atmosphere (CRISTA), Global Positioning System/Meteorological Experiment (GPS/MET), Limb Infrared Monitor of the Stratosphere (LIMS) and MLS. Preusse et al. (2000) showed that all four instruments provide largely consistent information on zonal mean temperature variances in the middle atmosphere if the observational filter of each instrument is approximated by a vertical visibility function, which is representative for the 300 to $800 \mathrm{~km}$ horizontal wavelength region. Good agreements when considering only one-dimensional filtering seem to imply that filtering of the horizontal wavelength is less important than filtering of the vertical wavelength.

As shown by Alexander (1998) and Preusse et al. (2000), global distributions of temperature variances may look very different depending on different observational filters. In particular, it was discussed whether all these measurements could be reliable when they exhibit large differences in the shape of the global distributions. The fact that applying the observational filter could explain these large differences among the various data sets emphasizes the importance of understanding the observational filter in a quantitative manner.

Another paper which clearly shows the important effect of the observational filter is that of Ern et al. (2005), in which the wavelength filtering was applied to GWMF provided by the Warner and McIntyre model (Warner and McIntyre, 2001) and an aliasing correction was applied to the CRISTA data. They showed that the agreement between GWMF observed by CRISTA and respective model values at an altitude of $25 \mathrm{~km}$ improved significantly after vertical wavelength filtering was applied. In particular, in terms of horizontal structure, most of the features shown by CRISTA observations were reproduced. Horizontal wavelength filtering modified horizontal distributions only slightly. However, it reduced GWMF magnitude by a factor of more than 2 .

In addition to infrared limb sounders, the impact of radiative transfer and retrieval was discussed also for other techniques. For instance, Wu and Waters (1997) showed the influence for MLS and Lange and Jacobi (2003) discussed GPS occultation measurements. A more general overview of observational filters for different instruments can be found in Preusse et al. (2008) and Alexander et al. (2010).

The publications mentioned above focus on the instrument visibility (effects of the radiative transfer). Gong et al. (2012) consider the filter for AIRS (Atmospheric Infrared Sounder) more carefully by taking into account the nadir observation geometry. In our current paper, we analyze for the first time a comprehensive observational filter for infrared limb sounders, which takes into account instrument visibility as well as observation geometry with a high level of accuracy. We show how such a comprehensive filter considerably affects the GW spectrum.

In our work, we applied the observational filter to a suitable model test case and investigated the effects of the observational filter on the shape of the modeled GWMF spectrum with respect to horizontal and vertical wave numbers (wavelengths). By spectral analysis, we demonstrated how various aspects of the observational filter affect GWs of different scales. For the test case, we used a combination of a convective gravity wave source (CGWS) scheme (Song and Chun, 2005, 2008) with the gravity wave regional or global ray tracer (GROGRAT; Marks and Eckermann, 1995; Eckermann and Marks, 1997) to generate GW distributions in the lower stratosphere at an altitude of $25 \mathrm{~km}$. The model generates a global distribution of individual waves, each fully characterized by location and a 3-D wave vector, thus forming a well-suited test case for our observational filter.

The CGWS scheme considers a diabatic forcing region in a three-layer atmosphere. The vertical structure of the forcing, which is a second-order polynomial, directly impacts the wave-filtering and resonance factor. This wave-filtering and resonance factor forms the spectral peaks in the momen- 
tum flux with respect to phase speed. Free tunable parameters of this scheme are the spatial and temporal scales of the diabatic forcing ( $\delta x$ and $\delta t$ ), which affect the horizontal wavelength as well as the phase speed. Different parameters for the CGWS scheme were considered. Parameter sets MF1 $(\delta x=5 \mathrm{~km}$ and $\delta t=20 \mathrm{~min})$ and MF2 $(\delta x=25 \mathrm{~km}$, $\delta t=60 \mathrm{~min}$ ) were introduced by Song and Chun (2005) and Choi et al. (2012), respectively. We introduce and investigate in this work an additional spectrum MF3 with a larger spatial scale $(\delta x=120 \mathrm{~km}$ and $\delta t=60 \mathrm{~min})$.

For the real atmosphere the prevailing horizontal wavelengths are unknown. From the generation mechanism, all discussed temporal and spatial scales (MF1, MF2 and MF3) are plausible. In the scope of the current paper, our aim is not trying to solve this question, but providing a tool for reliably estimating whether such waves are visible and, most importantly, for quantitatively determining to which extent they are visible. It should be further noted that here we use the convective source model and the various parameter sets as only one example for the application of the filter. Our aim in this paper is to show how the different steps of the observational filter act on different wavelength scenarios and which steps are the most important ones independent of the scenario chosen. In particular, we will demonstrate how the observational filter affects both magnitude and the shape of the spectral distribution.

In our efforts to understand the distribution measured by a certain instrument in the current paper we should keep in mind that our ultimate aim in general is to determine the real world GWMF distribution. In previous studies (Ern et al., 2006; Orr et al., 2010; Geller et al., 2013) we primarily learned about the general shape of the global distribution. In particular, in Geller et al. (2013) substantial differences among models and measurements are found, which remain inconclusive; however, the error of gravity wave momentum flux (GWMF) is estimated to be a factor of $\sim 2-5$ (Ern et al., 2004), chiefly because of the observational filter effects described in the current work. From climate modeling studies, on the other hand, a knowledge of substantial better than a factor of 2 is requested (Sigmond and Scinocca, 2010). That is an apparent clash of what we need for climate studies and what we can provide by measurements. Application of the observational filter is one way out of this dilemma; we cannot reconstruct the true GWMF from the measurements more accurately, because there are too many unknowns in the true distribution. However, assuming we know the true distribution, we can calculate with much higher accuracy what we should observe, provided we have a sufficiently accurate description of the observational filter.

What does that mean? Our aim is a GCM with realistic GWMF, either resolved or parameterized. If we are to compare the full modeled GWMF directly with the observations, we never can reach the required accuracy. If we apply the observational filter to the model first, we can reach the accuracy, provided (a) the filter is sufficiently accurate and (b) a sufficiently large part of the spectrum is visible. Even if we are only able to falsify, this allows to tell whether a model is inaccurate and hence we can initiate improvements. We still do not have the true distribution, but we can definitely rule out incorrect ones and the form of the discrepancy may give us guidance how improvement may be achieved.

This makes the comprehensive observational filter for IR limb sounders so important: IR limb sounders cover a relatively large part of the GW spectrum (see condition $b$ ) and the observational filter needs to be comprehensive, because only a comprehensive filter will be accurate (see condition a).

Other types of satellite instruments have different observational filters. These techniques and whether they could be approached applying the methods described in this work, is described in Appendix A. The observational filter designed in this work describes the inevitable effects of the limb sounding technique and the modification of the spectral shape. It does not include errors either from the instrument (e.g., noise) nor potentially caused by the analysis method. The delineation from such effects is discussed in Appendix B.

The paper is organized as follows: instruments and their observation geometries are described in Sect. 2, global gravity wave simulations are presented in Sect. 3. The observational filter with different processes is described in detail and is applied to a spectrum from MF1 in Sect. 4. Further results of applying this observational filter to MF2 and MF3 as well as the quantification of GWMF reduction are outlined in Sect. 5. In Sect. 6, conclusions are given.

\section{Instruments and observation geometry}

\subsection{Limb-sounding technique}

Infrared limb sounding from satellites is a well-established method for exploring the middle atmosphere (Bailey and Gille, 1986; Gordley et al., 1994; Marshall et al., 1994; Riese et al., 1999; Preusse et al., 2002). The basic geometry of limb sounding is depicted in Fig. 1. The instrument looks from its orbit towards the Earth's horizon, through the atmosphere and into cold space. Three exemplary lines of sight (LOS) are depicted in Fig. 1 by green dashed lines. The radiance measured by the instrument results from emission and reabsorption along the LOS. For optically thin emissions, reabsorption is weak and most of the radiance stems from the region around the tangent point (purple dots), where the LOS is closest to the Earth's surface. For this case, radiative transfer can be described by a Gaussian weighting function (Preusse et al., 2002, 2008) centered around the tangent point and, accordingly, measurements are associated with the tangent altitude (blue arrow) and the location of the tangent point. The precise viewing geometry varies for the individual instruments. 


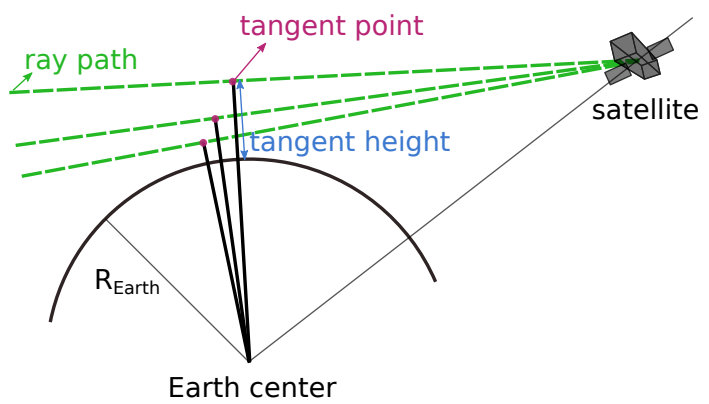

Figure 1. Measuring geometry of the limb-sounding technique.

\subsection{SABER instrument}

The SABER instrument uses broadband radiometers to detect limb radiance in the thermal infrared. Temperature is retrieved from the main $\mathrm{CO}_{2} \nu_{2}$ emission at $15 \mu \mathrm{m}$ (Remsberg et al., 2008). SABER was launched on 7 December 2001 onboard the TIMED (Thermosphere Ionosphere Mesosphere Energetics Dynamics) satellite into an orbit at an altitude of $625 \mathrm{~km}$ and inclination of $74.1^{\circ}$ and is still in operation.

The angle between flight direction and LOS, called "view angle" below, is schematically shown in Fig. 2. It alternates between $90^{\circ}$ for northward-looking mode and $270^{\circ}$ for southward-looking mode in yaw maneuvers roughly every 60 days. In Fig. 2, the black arrow shows the flight direction, the green line (SABER-N) indicates the LOS of SABER in the northward-looking mode, while the red line (SABER-S) is the LOS in the southward-looking mode. The corresponding latitude coverage of northward- and southward-looking modes changes between $52^{\circ} \mathrm{S}$ to $83^{\circ} \mathrm{N}$ and $83^{\circ} \mathrm{S}$ to $52^{\circ} \mathrm{N}$. More detailed information about the SABER instrument can be found, for instance, in Mlynczak (1997) and Russell III et al. (1999).

The orbital track and flight direction as well as satellite positions and corresponding tangent points for a typical southward-looking orbit of SABER are shown in Fig. 3a. Note that SABER views across the pole for the southern turning point. In Fig. 3a, green dots are the satellite positions, red triangles are the corresponding tangent points. Blue arrows along the satellite track show the flight direction, while the purple solid line indicates an example of a LOS. In addition, the latitude coverage during the year 2008 is shown in Fig. 4. Orange bands are coverages of the northward-looking mode, while blue bands indicate coverages of the southwardlooking mode.

\subsection{HIRDLS instrument}

The HIRDLS (High Resolution Dynamics Limb Sounder) instrument is an infrared radiometer onboard the Aura satellite, which also measures thermal emissions from the atmospheric limb. The orbit altitude and orbit inclination of Aura are $710 \mathrm{~km}$ and $98.2^{\circ}$, respectively. The HIRDLS instrument

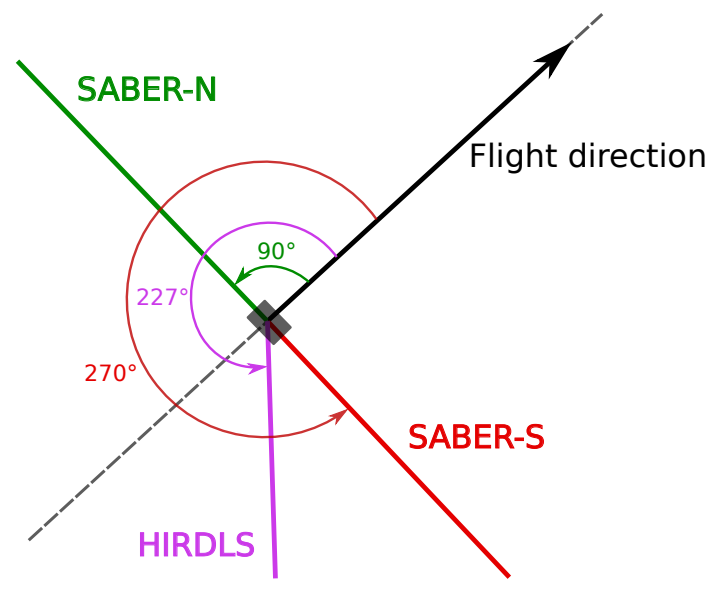

Figure 2. Satellite top-view of the SABER and HIRDLS viewing geometry, the black arrow shows the flight direction, green and red lines are the lines of sight (LOS) of SABER for northward- and southward-viewing modes, respectively. The purple line is the LOS of HIRDLS. For details, see text.

has a fixed view angle of $180+47=227^{\circ}$, which leads to a latitude coverage from about $63^{\circ} \mathrm{S}$ to about $80^{\circ} \mathrm{N}$. More detailed information about the HIRDLS instrument can be found, for instance, in Gille et al. (2003, 2008).

The view angle of HIRDLS is schematically depicted in Fig. 2 where the purple line illustrates the LOS of the HIRDLS instrument. In addition, satellite positions (green dots) and corresponding tangent points (red triangles) for an exemplary orbit are shown in Fig. 3b. HIRDLS's flight direction is indicated by blue arrows and the purple solid line shows an exemplary LOS.

\subsection{Observation geometry in the local coordinate system}

Our aim is to apply an observational filter to a simulated GW at a specific location. So far, we have seen in Fig. 2 the viewing geometry of SABER and HIRDLS with respect to the satellite. Now, in order to apply the observational filter, we need to determine the observation geometry with respect to the same local geophysical coordinate system in which the wave vector of the simulated GW is given. In Fig. 5, such an observation geometry is displayed for a short orbit segment. The instrument views in the direction of the LOS (blue solid arrows). The tangent points (blue crosses) are interpreted as the actual locations of the observations. The track of the tangent points, i.e., the track of the observations, is indicated by the green arrow. At one of the tangent points, a local coordinate system is shown (red axes). The angle between the LOS and the $x$ direction of the local coordinate system is called $\beta$ and the angle measured from the $x$ direction to the tangent-point track is called $\gamma$. Dependences of the angles $\beta$ and $\gamma$ on latitude for the observation geometry of SABER and HIRDLS are shown in Appendix C. 

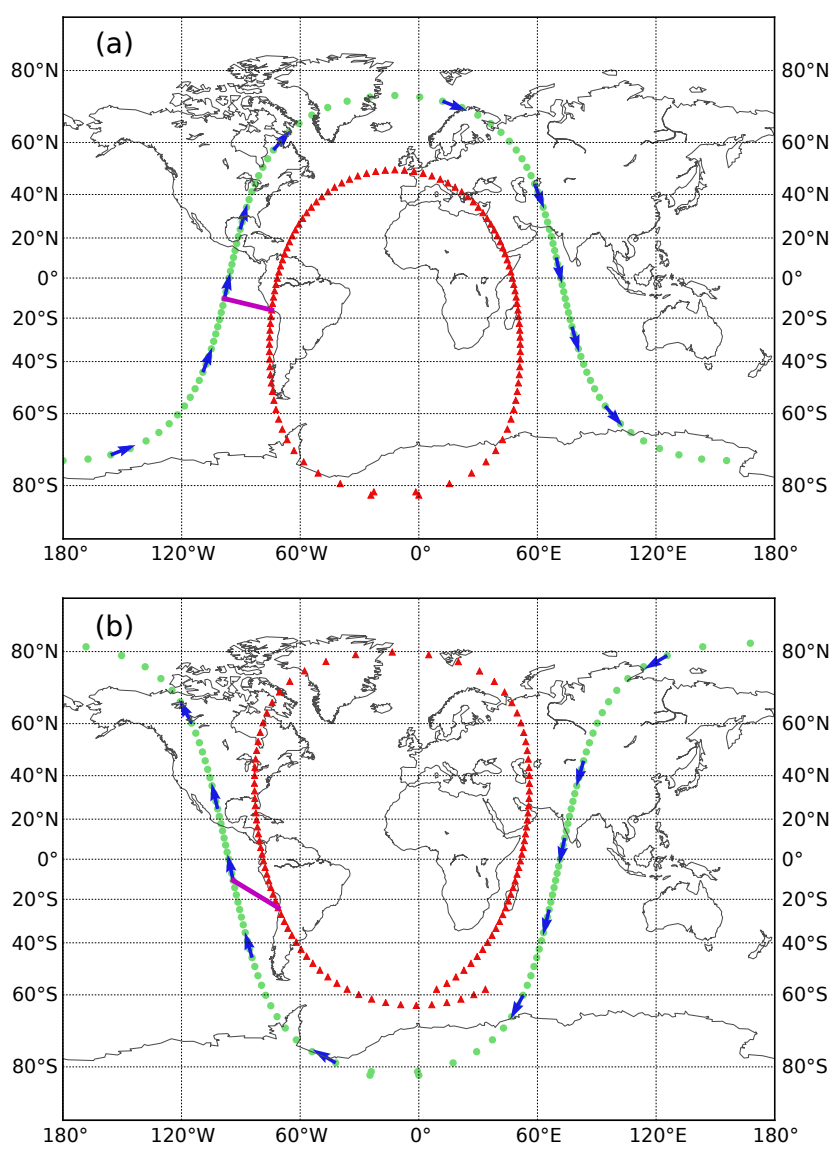

Figure 3. Global observation geometry of an exemplary orbit of (a) SABER and (b) HIRDLS. Satellite positions are shown by green dots and corresponding tangent points by red triangles. The thick purple line represents an exemplary LOS, while blue arrows show the flight direction. For details, see text.

\section{Global gravity wave simulation}

In order to demonstrate the application of the observational filter, we need a modeled GW distribution. Here, we use exemplarily ray-tracing simulations based on convective sources. Offline simulation of global gravity waves was performed by coupling the convective GW source (CGWS) scheme (Song and Chun, 2005) and the gravity wave regional or global ray tracer (GROGRAT) (Marks and Eckermann, 1995; Eckermann and Marks, 1997).

The CGWS scheme is formulated by applying a double Fourier transform in space and time to the perturbation solution of the primitive equations. The analytical model assumes a diabatic forcing region in a three-layer atmosphere. The vertical structure of the forcing is a second-order polynomial. This vertical structure directly impacts the wave-filtering and resonance factor, which in turn, forms the spectral peaks in the momentum flux with respect to phase speed. Calculation of this phase speed spectrum of GWMF requires the following quantities: maximum magnitude of the diabatic forcing

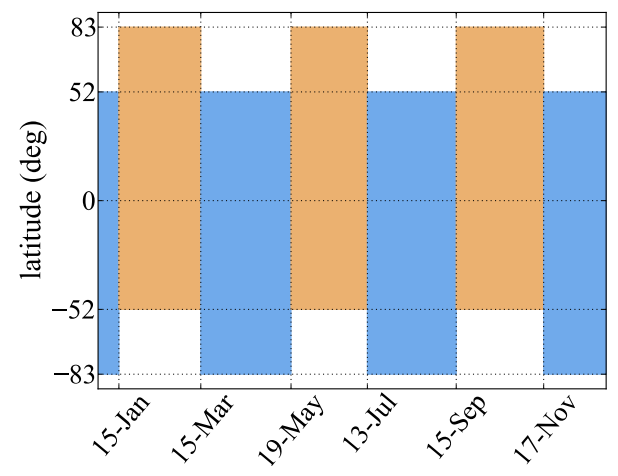

Figure 4. SABER latitude coverage during 2008; orange bands are coverages of northward viewing, while blue bands show coverages of southward viewing. For details, see text.

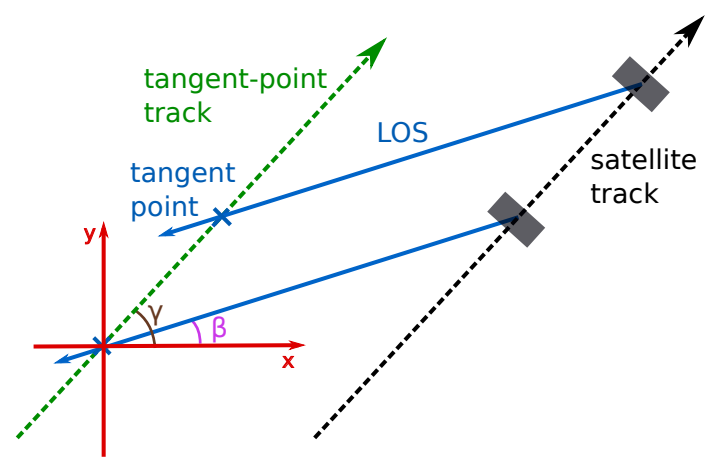

Figure 5. Satellite observation geometry in the local coordinate system in the two-dimensional horizontal plane. The black dashed line indicates the satellite track, while the green dashed line shows the tangent-point track. Blue lines are LOS. Red axes represent the local coordinate system. For details, see text.

$\left(q_{0}\right)$; bottom level $\left(z_{\mathrm{b}}\right)$ and top level $\left(z_{\mathrm{t}}\right)$ of the diabatic forcing; and moving speed of the diabatic forcing $\left(c_{q}\right)$. The first three quantities were taken from latent heat data of 3-hourly MERRA (modern-era retrospective analysis for research and applications) assimilated data for January 2008. The fourth is taken from the wind profile of MERRA data. The vertical structure and phase speed of the GWs induced by the diabatic forcing is influenced by the wave-filtering and resonance factor. The MERRA data were also used to provide background wind and temperature fields for our GROGRAT simulations. More detailed information about MERRA data as well as convective parameterization in MERRA can be found, for instance, in Rienecker et al. (2011), Kim and Alexander (2013) and Wright and Fueglistaler (2013).

Two free parameters of the parameterization are the spatial and temporal scales $(\delta x$ and $\delta t)$ of the diabatic forcing. We considered three different sets of $\delta x$ and $\delta t$, namely MF1 $(\delta x=5 \mathrm{~km}$ and $\delta t=20 \mathrm{~min}), \operatorname{MF} 2(\delta x=25 \mathrm{~km}$ and 
$\delta t=60 \mathrm{~min})$ and MF3 $(\delta x=120 \mathrm{~km} \text { and } \delta t=60 \mathrm{~min})^{1}$. The combination of MF1 and MF2 showed good agreement in spatial distribution as well as magnitude with AIRS observations (Choi et al., 2012). However, it is unable to explain the spectral peaks found by Ern and Preusse (2012). A possible reason is that MF1 and MF2 do not describe the presence of convective clusters, which could be represented by MF3.

In order to obtain spectral distributions in terms of horizontal and vertical wave numbers (wavelengths), GWMF with corresponding horizontal and vertical wave numbers were calculated directly from the ray-tracing simulation for an altitude of $25 \mathrm{~km}$. We considered global means, but took into account the latitude coverage of satellite instruments, which were mentioned in Sect. 2. It should be mentioned that, although the global mean is taken, the resulting spectrum will be dominated by the tropics and subtropics, because the dominant convective GW sources are located there. The respective simulated GWMF (symbolized by $F$ ) values were then binned according to horizontal and vertical wave numbers $\left(k_{\mathrm{h}}\right.$ and $m$ ) using a technique similar to that of Ern and Preusse (2012). All spectra were plotted in a base 10 logarithmic scale; i.e., $\widetilde{k}_{\mathrm{h}}=\log _{10}\left(1 / \lambda_{\mathrm{h}}\right)$ and $\widetilde{m}=\log _{10}\left(1 / \lambda_{z}\right)$, where $\lambda_{\mathrm{h}}$ and $\lambda_{z}$ are the horizontal and vertical wavelengths, respectively. The size of each bin was set as $\delta \widetilde{k_{\mathrm{h}}}=0.1$ and $\delta \widetilde{m}=0.1$. The simulated spectral distribution is called "true spectral distribution" (or "true spectrum"), because this would be the atmospheric spectrum if the model were to accurately represent the real atmosphere. In the following sections, we will discuss how this contrasts to a spectrum that would be observed by an infrared limb sounder. An example of the true spectrum for January 2008 for the parameter set MF1 is shown in Fig. 6a.

\section{Observational filter}

Based on the convective model and parameter settings for MF1, MF2 and MF3 described in Sect. 3 GWMF spectra

\footnotetext{
${ }^{1}$ Convective parameterizations comprehend a simplified physical description of the entire dynamics of a convective system and provide only the net effects to the general circulation model. They do not provide explicit information on, e.g., the spatial scale or on the moving-speed of clouds which are therefore important free parameters of the CGWS scheme (the moving speed in terms of a representative height; for this height, the background winds are assumed to drive the moving speed). For MF1 and MF2, the assumed spatial scales $\delta x$ are much smaller than a typical GCM grid distance, and we have a physical consistent picture of two subgrid parameterizations. The picture is less consistent, though, if the assumed size of the convective system $\delta x$ exceeds the grid spacing of the GCM. Still, such choices may be necessary, if the global GW distribution shall be solely described by the ray-tracer, or if due to missing dynamical feedback between the convection parameterization and the GCM dynamical core such waves are not generated in the model (Preusse et al., 2014). In this case they would need to be parameterized even if the model in principle is able to resolve the waves.
}

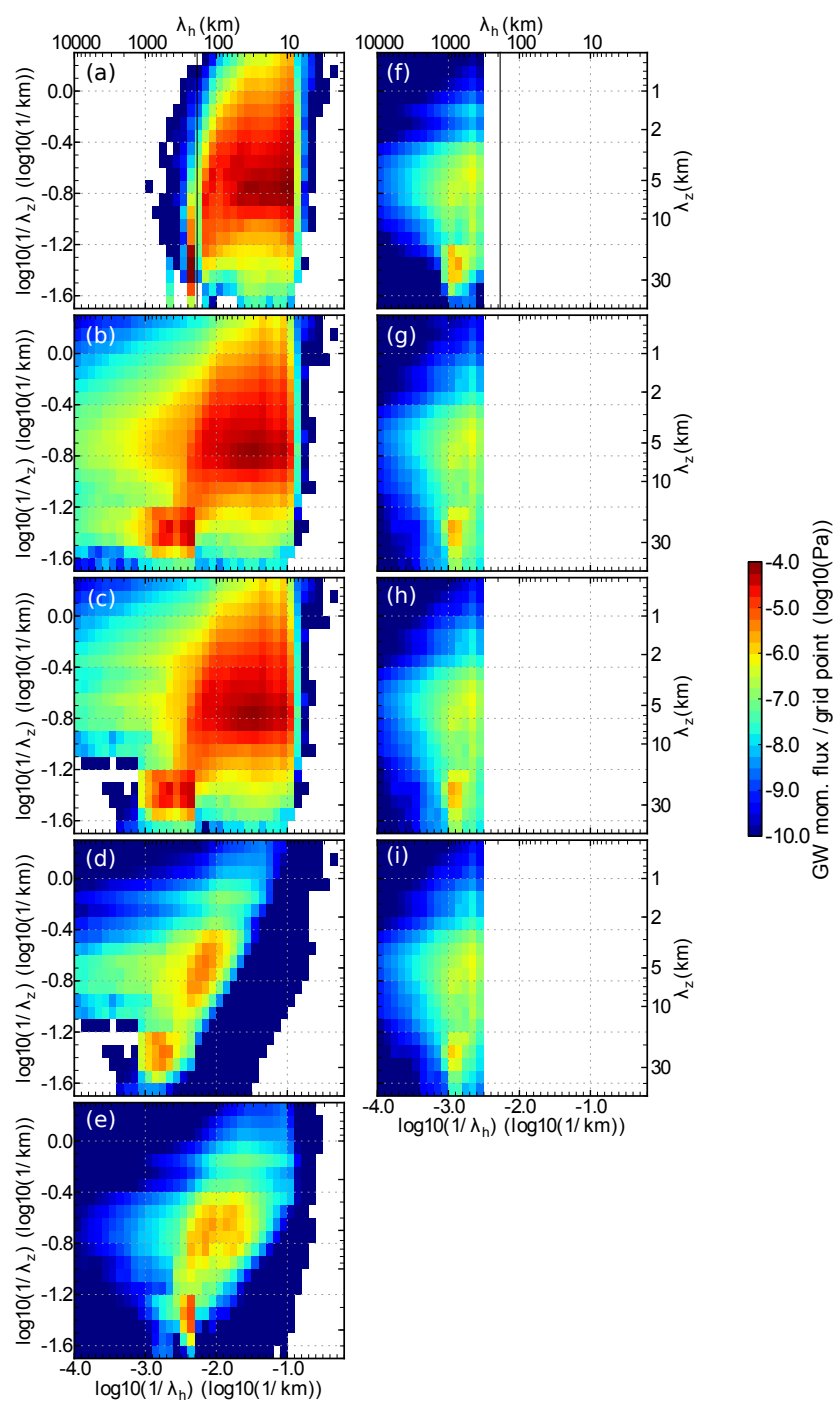

Figure 6. Spectral distributions of MF1 through different steps of the observational filter for January 2008 with the observation geometry of SABER, where (a) is the true spectrum, (b) along-LOS spectrum, (c) $\lambda_{\mathrm{h}}$ restriction spectrum, (d) instrument-sensitivity spectrum, (e) projection-on-track spectrum, (f) aliasing-effect spectrum, (g) $\lambda_{z}$, obs spectrum, (h) $\lambda_{z}$ restriction spectrum, (i) observed spectrum (after the additional correction). Black vertical lines in (a) and (f) indicate $\lambda_{\mathrm{h}}=185 \mathrm{~km}$. For details, see text.

were generated. In this section, we outline how an infrared limb sounder would observe these spectra; i.e., these spectra serve as reference for the influence of the observational filter. For short we will call these spectra therefore "true spectra" where "true" refers only to not being modified by any observational effects. The application of the comprehensive observational filter comprises four main processes. Each process is explained in one of the following subsections. The effects of each of these processes are shown in Fig. 6 by applying the observational filter for the observation geometry of the SABER instrument to the spectral distribution from 
(a)

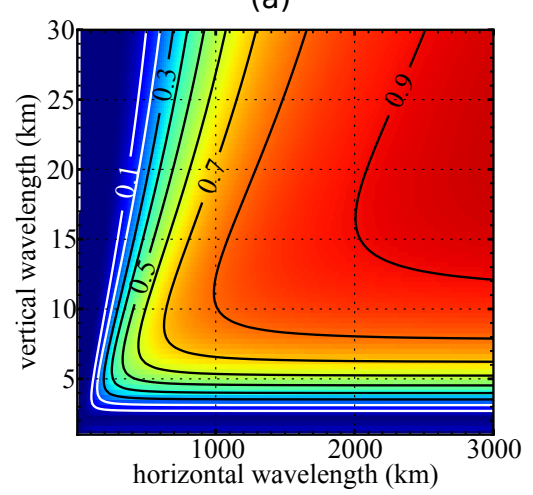

(b)

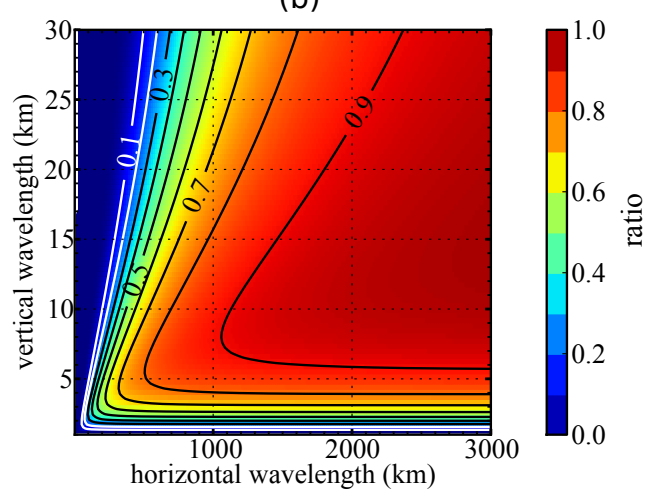

Figure 7. Two-dimensional sensitivity function for GWMF of (a) SABER and (b) HIRDLS.

MF1. The reason for choosing MF1 and SABER is that MF1 has the shortest spatial scale among the three parameterized spectra and that SABER has a longer sampling distance than HIRDLS. The effects of the filter on the GWMF spectrum are therefore most pronounced in this case.

\subsection{Visibility filter}

First, we consider the effects due to radiative transfer and retrieval, which also limit the waves that are visible to the instrument. We use an analytical approximation of the 2-D visibility filter for infrared limb sounding, which was derived by Preusse et al. (2002). This filter is based on two-dimensional cross sections through quasi-monochromatic waves. Preusse et al. (2002) assumed that all LOSs of a given profile form a two-dimensional plane, consisting of the vertical and one horizontal axis in the viewing direction of the instrument. The similar approach was also applied for analyzing the visibility of gravity waves measured by radio occultation in the paper of Lange and Jacobi (2003).

Following the analytical approach of Preusse et al. (2002), the instrument sensitivity of infrared limb sounders for temperature amplitude is

$S=\frac{\lambda_{z} \sqrt{2}}{2 \pi \Delta_{z}} \sqrt{1-\cos \left(\frac{2 \pi \Delta_{z}}{\lambda_{z}}\right)} \exp \frac{-c b^{2}}{4\left(c^{2}+a^{2}\right)}$,

where $\quad a=m / 2 R_{\mathrm{E}}=\pi /\left(\lambda_{z} R_{\mathrm{E}}\right), \quad b=k_{\mathrm{h}}=2 \pi / \lambda_{\mathrm{h}}$, $c=1 /\left(2 \mathrm{HR}_{\mathrm{E}}\right), R_{\mathrm{E}}$ is the Earth's radius, $H$ scale height, $k_{\mathrm{h}}$ horizontal wave number, and $m$ vertical wave number. The values of $R_{\mathrm{E}}$ and $H$ are 6350 and $6.5 \mathrm{~km}$, respectively. The vertical resolution $\Delta z$ is $2 \mathrm{~km}$ for SABER and $1 \mathrm{~km}$ for HIRDLS.

As shown by Ern et al. (2004), GWMF can be deduced from the temperature amplitude of the wave as follows:

$F=\frac{1}{2} \rho \frac{k_{\mathrm{h}}}{m}\left(\frac{g}{N}\right)^{2}\left(\frac{\hat{T}}{T}\right)^{2}$, where $\rho$ is the background atmosphere density, $g$ the gravity acceleration, $N$ the buoyancy frequency, $T$ the background temperature and $\hat{T}$ is the temperature amplitude of the wave. The sensitivity function $\sigma$ for GWMF, according to Eq. (2), is therefore obtained by squaring the temperature amplitude ratio:

$\sigma=S^{2}$.

Figure 7 illustrates the sensitivity function $\sigma$ for GWMF from (a) SABER and (b) HIRDLS. Comparing these two sensitivities, it is evident that HIRDLS has higher sensitivity owing to its higher vertical resolution, especially at short vertical wavelengths. For HIRDLS, a reasonable sensitivity (0.3) can be found down to a vertical wavelength of about $2 \mathrm{~km}$, whereas for SABER, this limit is approximately $3.5 \mathrm{~km}$. Sensitivities of the two instruments in the horizontal direction are comparable.

The visibility function is a function of two variables: the vertical wavelength and the projection of the horizontal wavelength onto the LOS (see below). Figure 8 combines the viewing geometry of the satellite with the geometry of the observed GW in the horizontal plane. In this figure, part of an exemplary wave is shown by the dashed grey curve. The red arrow indicates the direction of the wave vector and purple lines indicate wave fronts. $\psi$ is the angle between the wave vector and the $x$ direction of the local coordinate system $(\psi=\arctan (l / k)$ where $k$ and $l$ are wave numbers in the $x$ and $y$ directions, respectively). The horizontal wavelength $\lambda_{\mathrm{h}}$ is shown by the two-headed arrow, which is perpendicular to the wave fronts and parallel to the wave vector. The horizontal wavelength along $\operatorname{LOS}\left(\lambda_{\mathrm{h}, \mathrm{LOS}}\right)$, on the other hand, is parallel to the LOS (blue line) and is, in general, longer than $\lambda_{\mathrm{h}}$. 


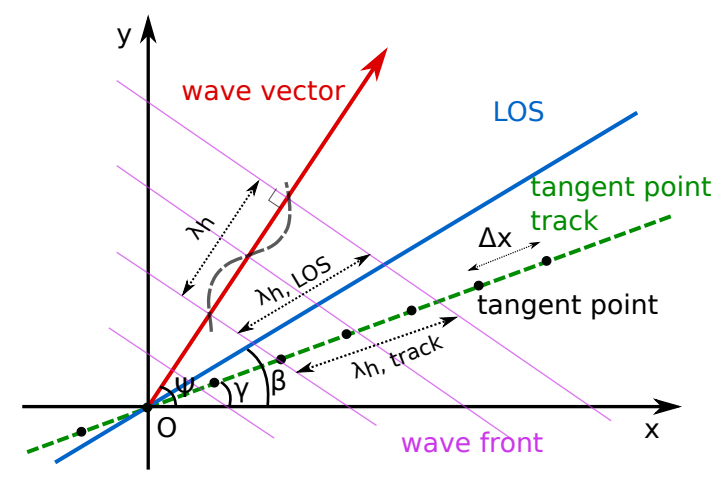

Figure 8. Combination of the satellite's viewing geometry and the geometry of the observed GW. One LOS (blue line) is shown for the tangent point at the origin. The horizontal wavelength along LOS $\left(\lambda_{\mathrm{h}, \text { LOS }}\right)$ can be calculated knowing the true horizontal wavelength $\left(\lambda_{\mathrm{h}}\right)$ and angles $\beta$ and $\psi$. The projection of horizontal wavelength on tangent-point track can be calculated knowing the true horizontal wavelength $\left(\lambda_{h}\right)$ and angles $\gamma$ and $\psi$. For details, see text.

Knowing $\lambda_{\mathrm{h}}$ and the angle $\beta$, the along-LOS horizontal wavelength $\lambda_{\mathrm{h}, \text { LOS }}$ can be calculated as follows:

$\lambda_{\mathrm{h}, \mathrm{LOS}}=\frac{\lambda_{\mathrm{h}}}{|\cos (\psi-\beta)|}$.

Figure $6 \mathrm{~b}$ shows the spectrum of $F$ with respect to $\lambda_{\mathrm{h}, \mathrm{LOS}}$ and $\lambda_{z}$. It is referred to as "along-LOS spectrum" hereafter. This spectrum, as we would expect, spreads in the direction of longer horizontal wavelengths.

The application of the visibility filter as described above assumes infinite plane wave fronts. However, three dimensional simulations of CGWs from single convective towers exhibit concentric wave fronts (Piani et al., 2000; Lane et al., 2001). The assumption therefore is clearly non-realistic, in particular for short period, short horizontal wavelength CGWs. This is problematic in cases where the horizontal wave vector is almost perpendicular to the horizontal LOS, the along-LOS wavelength approaches infinity, and the wave would therefore be regarded as visible. However, in a three-dimensional consideration, the LOS would still intersect many wave fronts resulting in a vanishing net signal. Thus, these waves should not be regarded as visible. In order to mask all waves which have short horizontal wavelengths but are only seemingly visible, we firstly introduce a "stretching" factor:

$\theta_{\mathrm{str}}=\frac{\lambda_{\mathrm{h}, \mathrm{LOS}}}{\lambda_{\mathrm{h}}}$,

and secondly, we simultaneously consider whether the horizontal wavelength is short compared to the shortest visible horizontal wavelength. Here, the shortest visible horizontal wavelength is determined as the value of $\lambda_{h}$ from Eq. (1) corresponding to a temperature sensitivity of 0.3 : $\lambda_{\text {vis }}=\lambda_{\text {vis }}\left(\lambda_{z}, S=0.3\right)$.

We also introduce the visibility ratio as

$\theta_{\text {vis }}=\frac{\lambda_{\mathrm{h}}}{\lambda_{\text {vis }}}$,

and threshold values of $\theta_{\text {str }}$ and $\theta_{\text {vis }}$ are denoted as $\theta_{\text {str, thresh }}$

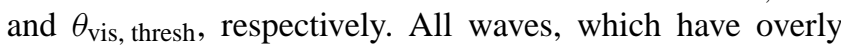
large stretching factors $\left(\theta_{\text {str }}>\theta_{\text {str, thresh }}\right)$ and simultaneously have overly short horizontal wavelengths $\left(\theta_{\text {vis }}<\theta_{\text {vis, thresh }}\right)$, are set to zero temperature amplitude as well as to a zero GWMF value. For this study, we chose $\theta_{\text {str, thresh }}=5$ and $\theta_{\text {vis, thresh }}=1$. As shown later, results are not very sensitive on the choice of these threshold values (cf. Fig. 15). This restriction was applied before the application of the instrument sensitivity function. GWMF values after considering this restriction are given by $F_{\text {restr }}$. A spectral distribution of $F_{\text {restr }}$ with respect to $\lambda_{\mathrm{h}, \operatorname{LOS}}$ and $\lambda_{z}$, which is called " $\lambda_{\mathrm{h}}$-restriction spectrum" hereafter, is shown in Fig. $6 \mathrm{c}$, again using $\lambda_{\mathrm{h}, \mathrm{LOS}}$ for the $x$ axis. Comparing with Fig. 6b, it can be seen that part of the spectral distribution at long $\lambda_{z}$ and long $\lambda_{\mathrm{h}, \operatorname{LOS}}$ is removed.

After this consideration of the horizontal wavelength restriction, the sensitivity function was applied to GWMF. An example of this application of the SABER sensitivity function on MF1 is shown in Fig. 6d. This spectrum is referred to as "instrument-sensitivity spectrum" hereafter. In comparison with the previous spectrum (Fig. 6c), it is clear that a significant part of the spectrum associated with short vertical and horizontal wavelengths has been filtered out. The area of high-value GWMF has now shifted to the direction of longer horizontal as well as vertical wavelengths. GWMF values after applying the sensitivity function are denoted as $F_{\text {vis }}$.

\subsection{Projection of the wavelength on the tangent-point track}

Today's limb scanning satellite instruments provide information only along track. Therefore, from current limb sounders only the projection of the horizontal wavelength on the tangent-point track can be estimated (Ern et al., 2004; Preusse et al., 2009b). The horizontal sampling of currentday satellite observations is too sparse to directly infer the horizontal wavelength. This problem is circumvented by first analyzing vertical profiles and determining vertical wavelengths, amplitudes and phases dependent on altitude. The horizontal wavelength is then estimated from the phase difference of adjacent profiles at the same altitude and the distance between observations along the tangent-point track. The method was first introduced by Ern et al. (2004). Although there are different applications with respect to the profile analysis (Alexander et al., 2008; Wright et al., 2010) they all rely on phase differences along the orbital track. In particular, if the phase difference is $\Delta \Phi$ and the sampling 
distance between two altitude profiles is $\Delta x$, the horizontal wave number and horizontal wavelength along the tangentpoint track $\left(k_{\mathrm{h} \text {, track }}\right.$ and $\left.\lambda_{\mathrm{h} \text {, track }}\right)$ can be estimated as follows:

$k_{\mathrm{h}, \text { track }}=\frac{\Delta \Phi}{\Delta x}=\frac{2 \pi}{\lambda_{\mathrm{h}, \text { track }}}$.

In our simulation, $\lambda_{\mathrm{h} \text {, track }}$ was calculated from the horizontal wavelength $\lambda_{h}$ based on the geometric relation between them. This geometric relation is illustrated also in Fig. 8. In this figure, black dots are tangent points, and the green dashed line shows the tangent-point track. The horizontal wavelength along the tangent-point track $\left(\lambda_{\mathrm{h}, \text { track }}\right)$ is indicated by the two-headed arrow, which is parallel to the tangent-point track. It is clear that the angle between the wave vector and the tangent-point track is $\psi-\gamma$. From here,

$\lambda_{\mathrm{h}, \text { track }}=\frac{\lambda_{\mathrm{h}}}{|\cos (\psi-\gamma)|}$.

Due to the projection, the horizontal wave number is changed in Eq. (2), and as GWMF and horizontal wave number are proportional, the momentum flux calculated from $\lambda_{\mathrm{h} \text {, track }}$ is

$\frac{F_{\text {track }}}{F_{\text {vis }}}=\frac{k_{\mathrm{h}, \text { track }}}{k_{\mathrm{h}}}=\frac{\lambda_{\mathrm{h}}}{\lambda_{\mathrm{h}, \text { track }}}$,

or

$F_{\text {track }}=F_{\text {vis }} \frac{\lambda_{\mathrm{h}}}{\lambda_{\mathrm{h}, \text { track }}}$.

A spectral distribution of $F_{\text {track }}$ in terms of $\lambda_{\mathrm{h} \text {, track }}$ and $\lambda_{z}$ is shown in Fig. 6e. This spectrum is called "projection-ontrack spectrum" hereafter and contains both the effects of visibility filtering and along-track projection.

\subsection{Aliasing effect}

\subsubsection{Calculation of horizontal wavelength due to the aliasing effect}

Satellite measurements are performed discretely which leads to a so-called aliasing effect, one of the well-known limitations of discrete sampling. The Nyquist theorem states that two samples per wave period or wavelength are necessary to properly resolve the wave. In other words, sampling distance $\Delta x$ of less than a half of $\lambda_{\mathrm{h} \text {, track }}$ is required to properly infer the wave structure from the observed data.

For SABER, $\Delta x=185 \mathrm{~km}$ was used as the sampling distance for our calculations. In the case of HIRDLS, $\Delta x$ is different for different operation periods. The shortest pair distance at the altitude of $25 \mathrm{~km}$ was about $70 \mathrm{~km}$ and we used $\Delta x=70 \mathrm{~km}$ for calculations of HIRDLS. More details about sampling distances of various satellite instruments can be found in Ern et al. (2011).

In order to estimate the horizontal wavelength caused by the aliasing effect $\left(\lambda_{\mathrm{h}}\right.$ alias $)$, we emulated the phasedifference method applied to the measurements. First, the

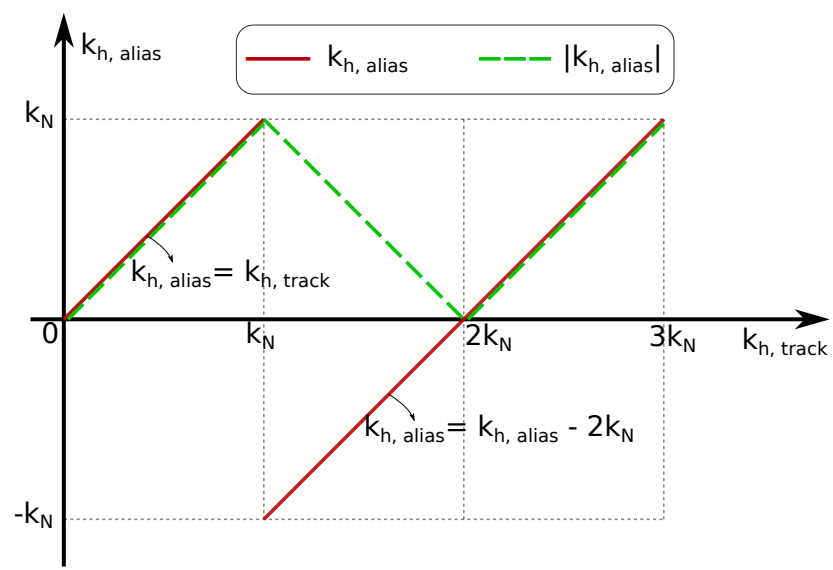

Figure 9. "Alias" wave number vs. wave number along tangentpoint track.

phase difference $\Delta \Phi$ between two adjacent vertical profiles is required. From Eq. (8), $\Delta \Phi$ can be defined as follows:

$\Delta \Phi=k_{\mathrm{h}, \text { track }} \Delta x=\frac{2 \pi \Delta x}{\lambda_{\mathrm{h}, \text { track }}}$.

Without further information, we had to assume that phase differences $\Delta \Phi$ are in the interval $[-\pi, \pi]$ despite the fact that the real phase differences may be larger. This is in accordance with the Nyquist theorem, where a phase difference larger than $\pi$ causes a wavelength shorter than the Nyquist wavelength, which is twice the sampling distance: $\lambda_{\mathrm{N}}=2 \Delta x$, where $\lambda_{\mathrm{N}}$ is the Nyquist wavelength.

Hence, in the current work, the phase difference $\Delta \Phi$ given by Eq. (12) was wrapped into interval $[-\pi, \pi]$. This wrapping process provided $\Delta \Phi_{\mathrm{wrap}} \in[-\pi, \pi]$ and the absolute value of the horizontal wave number due to aliasing effect $\left(k_{\mathrm{h}, \text { alias }}\right)$ can be calculated as follows:

$\left|k_{\mathrm{h}, \text { alias }}\right|=\frac{\left|\Delta \Phi_{\mathrm{wrap}}\right|}{\Delta x}$.

The dependence of $k_{\mathrm{h} \text {, alias }}$ and $\left|k_{\mathrm{h} \text {, alias }}\right|$ upon $k_{\mathrm{h} \text {, track }}$, for instance, in the interval $\Delta \Phi \in[0,3 \pi]$, is illustrated in Fig. 9. Here $k_{\mathrm{N}}$ is the Nyquist limit of horizontal wave number:

$k_{\mathrm{N}}=\frac{\pi}{\Delta x}$.

Using the wrapped phase difference, $\lambda_{\mathrm{h} \text {, alias }}$ can be defined:

$\lambda_{\mathrm{h}, \text { alias }}=\frac{2 \pi}{\left|k_{\mathrm{h}, \text { alias }}\right|}=\frac{2 \pi \Delta x}{\left|\Delta \Phi_{\mathrm{wrap}}\right|}$.

\subsubsection{Calculation of GWMF corresponding to $\lambda_{h \text {, alias }}$}

In analogy to the deduction of Eq. (2), the relation between $F_{\text {track }}$ and GWMF corresponding to the aliased horizontal wavelength $\left(F_{\text {alias }}\right)$ is 
$\frac{F_{\mathrm{alias}}}{F_{\text {track }}}=\frac{k_{\mathrm{h}, \text { alias }}}{k_{\mathrm{h}, \text { track }}}=\frac{\lambda_{\mathrm{h}, \text { track }}}{\lambda_{\mathrm{h}, \text { alias }}}$,

or

$$
F_{\text {alias }}=F_{\text {track }} \frac{\lambda_{\mathrm{h}, \text { track }}}{\lambda_{\mathrm{h}, \text { alias }}} .
$$

The spectral distribution of $F_{\mathrm{alias}}$ with respect to $\lambda_{\mathrm{h} \text {, alias }}$ and $\lambda_{z}$ is hereinafter referred to as the "aliasing-effect spectrum", and the aliasing-effect spectrum for MF1, January 2008 is shown in Fig. 6f. In comparison with the spectrum of the previous step (Fig. 6e), a notably large part of the spectral distribution is cut off and flipped to the left, i.e., to longer horizontal wavelengths. The cut-off part is associated with horizontal wavelengths shorter than the Nyquist wavelength of $2 \Delta x=370 \mathrm{~km}$. Some GWMF is added to the left part of the spectrum, at wavelengths corresponding to aliased hori-

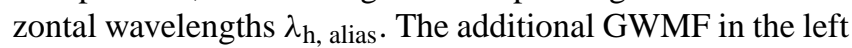
part is according to Eq. (17) smaller than the original GWMF on the right-hand side of Fig. 6e since $\lambda_{\mathrm{h} \text {, alias }}$ is longer than $\lambda_{\mathrm{h} \text {, track }}$ for these waves. In this aliasing-effect spectrum of MF1, artificial peaks were caused by the aliasing effect at horizontal wavelengths of about $800 \mathrm{~km}$. Overall, the magnitude of GWMF was reduced notably.

\subsection{Calculation of observed vertical wavelength}

Altitude profiles sampled by most limb sounders are nonvertical, which is an effect that also has to be considered. For example, for SABER and HIRDLS this applies and the effect is investigated and taken into account in our simulations. In particular, we calculate the vertical wavelength, which would be observed by the satellite instrument. This wavelength is referred to as observed vertical wavelength hereafter.

From observations, the vertical wavelength is derived by analyzing altitude profiles as provided by the instrument teams. It is generally assumed that these altitude profiles are vertical and that therefore only the vertical wave structure contributes to the wave structure in the profile. However, for SABER and HIRDLS, scans are not strictly vertical: The change in altitude is performed by upward and downward scanning by the instrument. However, during upward and downward scanning, the satellite moves along its track. This leads to a slant of the profile in the direction along the tangent-point track. Also, when the LOS moves up (down), the tangent-point becomes closer to (further from) to the satellite (cf. Fig. 1). This leads to another slant of the profile in the direction across the tangent-point track. Because of the slant of the altitude profiles, it can happen that during an altitude scan not only the vertical structure of an observed wave is sampled, but also to some extent the horizontal structure.

In Fig. 10, two exemplary tangent points $O_{1}, O_{2}$ along an altitude profile are illustrated (purple dots). This could correspond to any pair of adjacent altitudes in a scan such as illustrated in Fig. 1. A local coordinate system at tangent point $O_{1}$

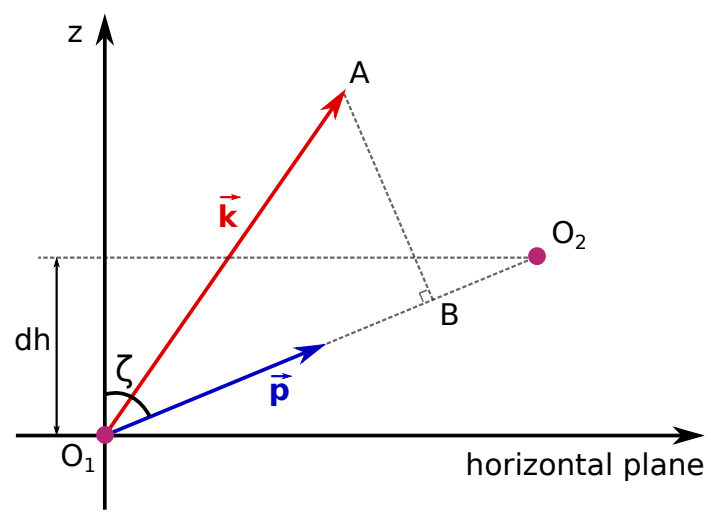

Figure 10. Observation geometry at a tangent point of an altitude profile. Two purple dots $\left(O_{1}\right.$ and $\left.O_{2}\right)$ represent two tangent points. The red arrow shows the wave vector, while the blue arrow is the normalized vector of the profile vector $\boldsymbol{O}_{1} \boldsymbol{O}_{2} . \mathrm{d} h$ is the altitude difference between $O_{1}$ and $O_{2}$.

is shown where the $z$ axis indicates the vertical direction. The altitude difference $\mathrm{d} h$ between two tangent points $O_{1}$ and $O_{2}$ is small (we chose $\mathrm{d} h=3 \mathrm{~km}$ ), so that the vector $\boldsymbol{O}_{1} \boldsymbol{O}_{2}$ was considered to be the local profile vector. If $\boldsymbol{p}$ (blue vector) is the normalized vector of $\boldsymbol{O}_{1} \boldsymbol{O}_{2}$ and $\boldsymbol{k}$ (red vector) is the wave vector, then the wave number along the profile can be defined as the scalar product of $\boldsymbol{k}$ and $\boldsymbol{p}$ :

$m_{\mathrm{p}}=\boldsymbol{k} \cdot \boldsymbol{p}$.

The wavelength along the profile is

$\lambda_{z, \mathrm{p}}=\frac{2 \pi}{m_{\mathrm{p}}}$.

From $\lambda_{z, \mathrm{p}}$ and from the angle $\zeta$ between the normalized profile vector $\boldsymbol{p}$ and the $z$ axis of the local coordinate system (cf. Fig. 10), the observed vertical wavelength $\lambda_{z}$, obs is calculated:

$\lambda_{z, \mathrm{obs}}=\lambda_{z, \mathrm{p}} \cos \zeta$

Momentum flux corresponding to this vertical observed

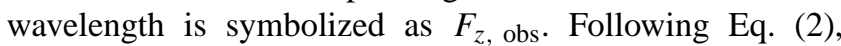
GWMF is inversely proportional to the vertical wave number and thus proportional to the vertical wavelength:

$F_{z, \text { obs }}=F_{\text {alias }} \frac{\lambda_{z, \text { obs }}}{\lambda_{z}}$.

In a statistical average, we will have as many upwardscanning observations as downward-scanning observations. Therefore, we calculate both solutions for each wave and show the average. The spectrum with observed vertical wavelength hereafter is referred to as " $\lambda_{z}$, obs spectrum" and an example for MF1 is shown in Fig. $6 \mathrm{~g}$. The spectrum was slightly redistributed towards longer vertical wavelengths. In 
particular, for vertical wavelengths longer than $6 \mathrm{~km}, \mathrm{GWMF}$ was slightly enhanced.

For every wave, we also examined the difference between the observed vertical wavelengths for the upward and downward scans. If this difference is greater than $40 \%$ of the average vertical wavelength, this wave will be rejected. We here follow the GWMF determination from real observations as described in Ern et al. (2011), where such pairs of profiles are not used by the momentum flux (MF) calculation method. It should be noted that for other methods of MF calculation, these pairs may be used.

We symbolize GWMF after this restriction as $F_{z}$, obs, restr. The spectrum with this restriction, called " $\lambda_{z}$-restriction spectrum" later, is shown in Fig. 6h. In comparison with the previous spectrum (Fig. 6g), only minor changes were found. In particular, the magnitude of GWMF surrounding the spectral peak at vertical wavelength of about $5 \mathrm{~km}$ was reduced slightly.

In the last step of the observational filter, we applied an additional correction, which was used in Ern et al. (2011). First, this correction removes dominant vertical oscillation of quasi-stationary planetary waves (which have a vertical wavelength $\geq 40 \mathrm{~km}$ ) in the altitude profiles. Second, it helps to keep only those vertical wavelengths for which amplitudes can reliably be determined in the $10 \mathrm{~km}$ vertical window of the Maximum Entropy Method/Harmonic Analysis (MEM/HA) spectral analysis (Preusse et al., 2002; Ern et al., 2011). The GWMF at this last step is denoted as $F_{\text {obs }}$.

This is the final step of our comprehensive observational filter. The resulting spectrum is therefore considered to represent the observed spectrum and is presented in Fig. 6i. In comparison with Fig. 6h, it can be seen that contributions of long vertical wavelength waves were somewhat reduced. However, the overall spectrum is changed only slightly.

A comparison of this observed spectrum and the true spectrum (Fig. 6a) shows that the spectral distribution of MF1 is significantly influenced by the observational filter in both shape and magnitude. In particular, the observed spectrum consists of horizontal wavelength for which MF1 did not generate any wave events and vice versa. This is due to the fact that MF1 has a small spatial scale and produces a large amount of short horizontal wavelength GWs, which can hardly be observed by limb sounders. However, as mentioned before, for demonstrating the different effects of the observational filter, MF1 was chosen because the different effects contributing to the observational filter can be demonstrated clearly. Later in the manuscript we will address other setups of the CGWS that produce wave spectra that can be better observed.

All steps of the observational filter are summarized by a flowchart in Fig. 11. The steps with significant changes are marked by bold characters. Additional examples of applying the observational filter to all three spectra MF1, MF2, MF3 using the observation geometries of SABER as well as HIRDLS will be presented in Sect. 5 below.

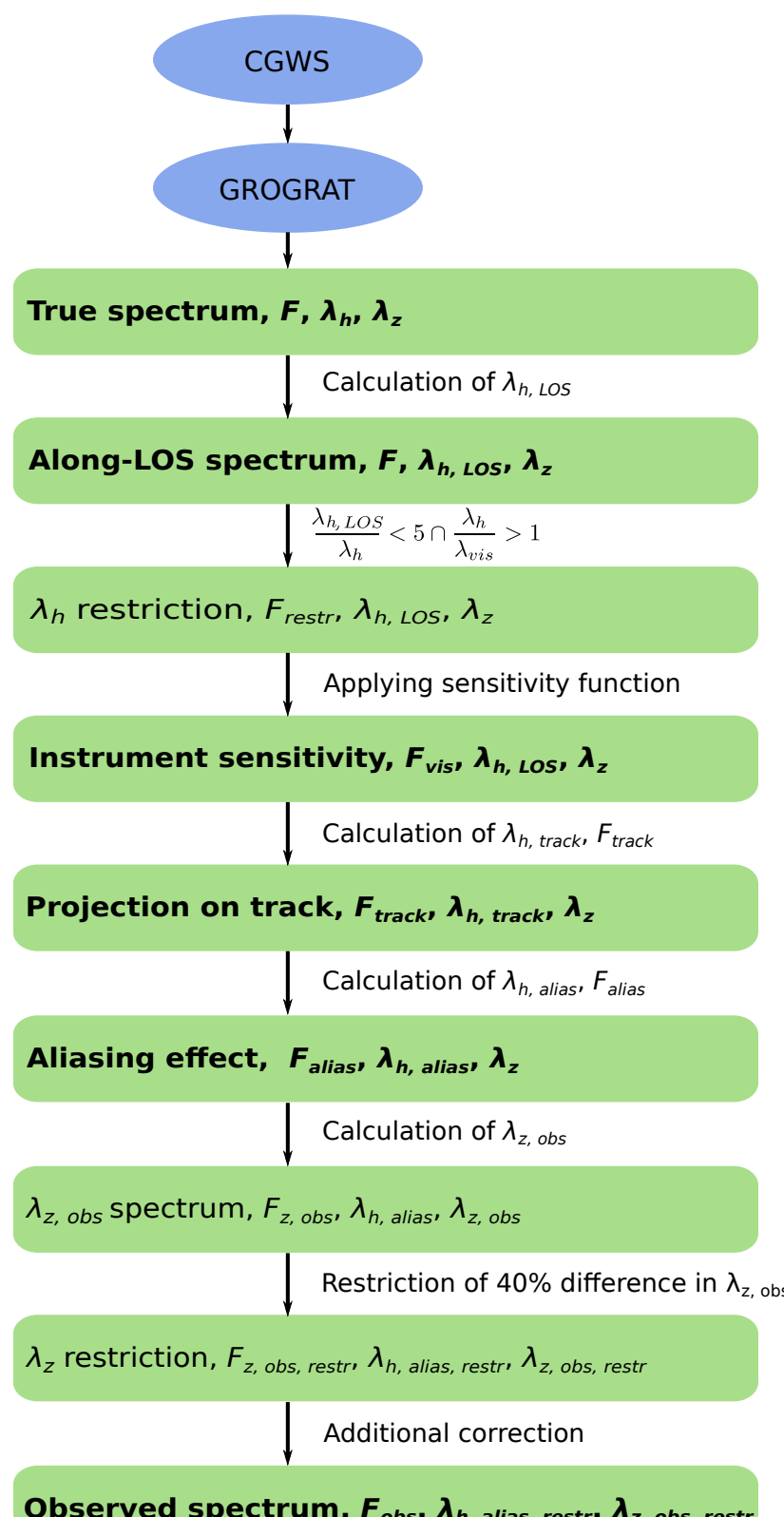

Observed spectrum, $\boldsymbol{F}_{\text {obs }}, \lambda_{h \text {, alias, restr, }} \lambda_{z \text {, obs, restr }}$

Figure 11. Overview of all steps the observational filter. The steps with significant changes are marked by bold characters.

\section{Further examples}

\subsection{Applying the observational filter to observation geometry of SABER}

In Sect. 4, we illustrated the observational filter by applying it to the spectrum of MF1 and using SABER geometry. In this section, we provide further examples by applying the observational filter to all spectra MF1, MF2 and MF3 and using observation geometry of both instruments (SABER and HIRDLS). 
For SABER geometry, the results of applying the observational filter are presented in Fig. 12. As shown by "true" simulated spectra (Fig. 12a, g, and m), MF3 provides GWs with the longest horizontal wavelength. The main spectral peak of MF3 is at a horizontal wavelength of about $220 \mathrm{~km}$. It has some sub-structure and extends to $\lambda_{\mathrm{h}}$ as high as few hundred $\mathrm{km}$. For MF2 and MF1, this peak is located at horizontal wavelengths of about 50 and $10 \mathrm{~km}$, respectively. It should be noted that the spatial scale of the cloud tower specified in the source model is imagined to act as a single body force without substructure. Therefore, no waves with wavelength of the order or shorter than this body force are excited. Instead, the model produces a sharp onset at the wavelength of twice this size. This sharp onset is pronounced at the source altitude. When GWs propagate upward, the wavelength may be modified by horizontal refraction (e.g., Marks and Eckermann, 1995) which slightly weakens this sharp onset. In the cases of MF1 and MF2, at the considered altitude of $25 \mathrm{~km}$, this sharp onset still can be seen quite clearly.

Due to this difference in the spatial scale, the observational filter affects MF1, MF2 and MF3 differently. For example, the effect of the $\lambda_{h}$ restriction (Sect. 4.1) on MF1 is recognizable by comparing Fig. $6 \mathrm{~b}$ and c, while for MF2 and MF3, this effect is minor and indicated by only an insignificant decrease in GWMF at long horizontal and vertical wavelengths (not shown).

However, differences can be seen much more clearly after the instrument sensitivity has been applied by comparing the second and third rows of Fig. 12. For MF1, a very large amount of GWMF corresponding to short horizontal as well as vertical wavelengths has been filtered out (cf. Fig. 12b and c). The spectral peak is shifted from a $\lambda_{h, L O S}$ value of about $40 \mathrm{~km}$ (Fig. 12b) to a value of about $160 \mathrm{~km}$ (Fig. 12c). It should be noted that in this step, spectra are plotted with respect to the horizontal wavelength along $\operatorname{LOS}\left(\lambda_{\mathrm{h}, \mathrm{LOS}}\right)$. The shift of the spectral peak with respect to the true horizontal wavelength $\left(\lambda_{h}\right)$ in general is shorter. For MF2, the reduction in GWMF is considerably smaller than for MF1 (cf. Fig. 12h and i). Nevertheless, the GWMF magnitude is reduced quite strongly. The spectral shape changes and the area of strong GWMF moves to the direction of longer horizontal and vertical wavelengths. For MF3, part of the spectrum related to short wavelengths has also been filtered out (cf. Fig. 12n and o). This part, however, is smaller than for MF2, and although GWMF magnitude has decreased, the main spectral peak of MF3 remains at the same position (at $\lambda_{\mathrm{h}, \mathrm{LOS}}$ of about $500 \mathrm{~km})$.

Figure $12 \mathrm{~d}, \mathrm{j}$, and $\mathrm{p}$ show spectra of $F_{\text {track }}$ with respect to $\lambda_{\mathrm{h} \text {, track }}$ and $\lambda_{z}$. For MF1 and MF2, GWs with a horizontal wavelength shorter than $100 \mathrm{~km}$ contribute quite strongly to the spectrum (cf. Fig. 12d and j). High values of GWMF are even found at horizontal wavelengths down to about 20$30 \mathrm{~km}$. In contrast, the main part of the spectrum of MF3 arises from by GWs with a horizontal wavelength greater than $100 \mathrm{~km}$ (cf. Fig. 12p). The influence of the aliasing ef- fect on MF3 is therefore weaker than on MF1 and MF2. This is shown in Fig. 12e, k, and q. Since MF1 and MF2 contain many more short horizontal-wavelength GWs, an essential part of their spectra is projected to the left. For MF1, the features of the spectrum are changed significantly, as described before in Sect. 4. For MF2, a strong alteration is also found, although no strong artificial spectral peaks appear, as they do in the case of MF1. In contrast, the part of MF3 projected to the left is minor in comparison with the originally long horizontal-wavelength part. Therefore, the strongest contribution to the spectrum in general, and the main peak in particular, still remains at the same position.

Figure 12f, 1, and $r$ shows observed spectra after the calculation of observed vertical wavelength, vertical wavelength restriction and additional correction. In comparison with aliasing-effect spectra, very minor changes were found for all spectra. In particular, spectra were redistributed slightly in the direction of longer vertical wavelengths, making them somewhat more homogeneous in this direction. The spectral peak at a vertical wavelength of about $30 \mathrm{~km}$ of MF1 was reduced in magnitude.

Briefly, the spectrum for MF3 was least influenced by the observational filter. For horizontal wavelengths longer than the Nyquist wavelength, major features were still conserved. The spectrum of MF1 was most influenced and significant changes were found in both shape and magnitude.

\subsection{Applying the observational filter to observation geometry of HIRDLS}

The observation geometry of HIRDLS has a shorter horizontal sampling distance. HIRDLS also has a higher vertical resolution. The results of applying the observational filter to the observation geometry of HIRDLS are presented in Fig. 13.

In the case of HIRDLS, "true" spectra (Fig. 13a, g, and m) are very similar to "true" spectra for SABER. Minor differences result from the different latitude coverage.

However, in contrast to SABER, along-LOS spectra of HIRDLS spread more strongly towards longer horizontal wavelengths (Fig. 13b, h, and $\mathrm{n}$ ). This is an effect of the average orientation of the simulated GWs with respect to different view angles of the two instruments. This effect depends not only on the differences in viewing geometry, but also on the simulated distribution of GWs.

The effects of the horizontal wavelength restriction were similar to those observed for SABER observation geometry with minor reductions at long horizontal and vertical wavelengths for all three spectra (not shown).

However, HIRDLS possesses better sensitivity to shortwavelength GWs, particularly in the vertical direction. This weakens the influence of the instrument's sensitivity to all spectra. A comparison of Figs. 12c, i, o and 13c, i, o shows that in comparison with SABER, for HIRDLS, the amount of GWMF was not reduced as much by the instrument sensitivity. For HIRDLS, GWMF was still conserved quite well 

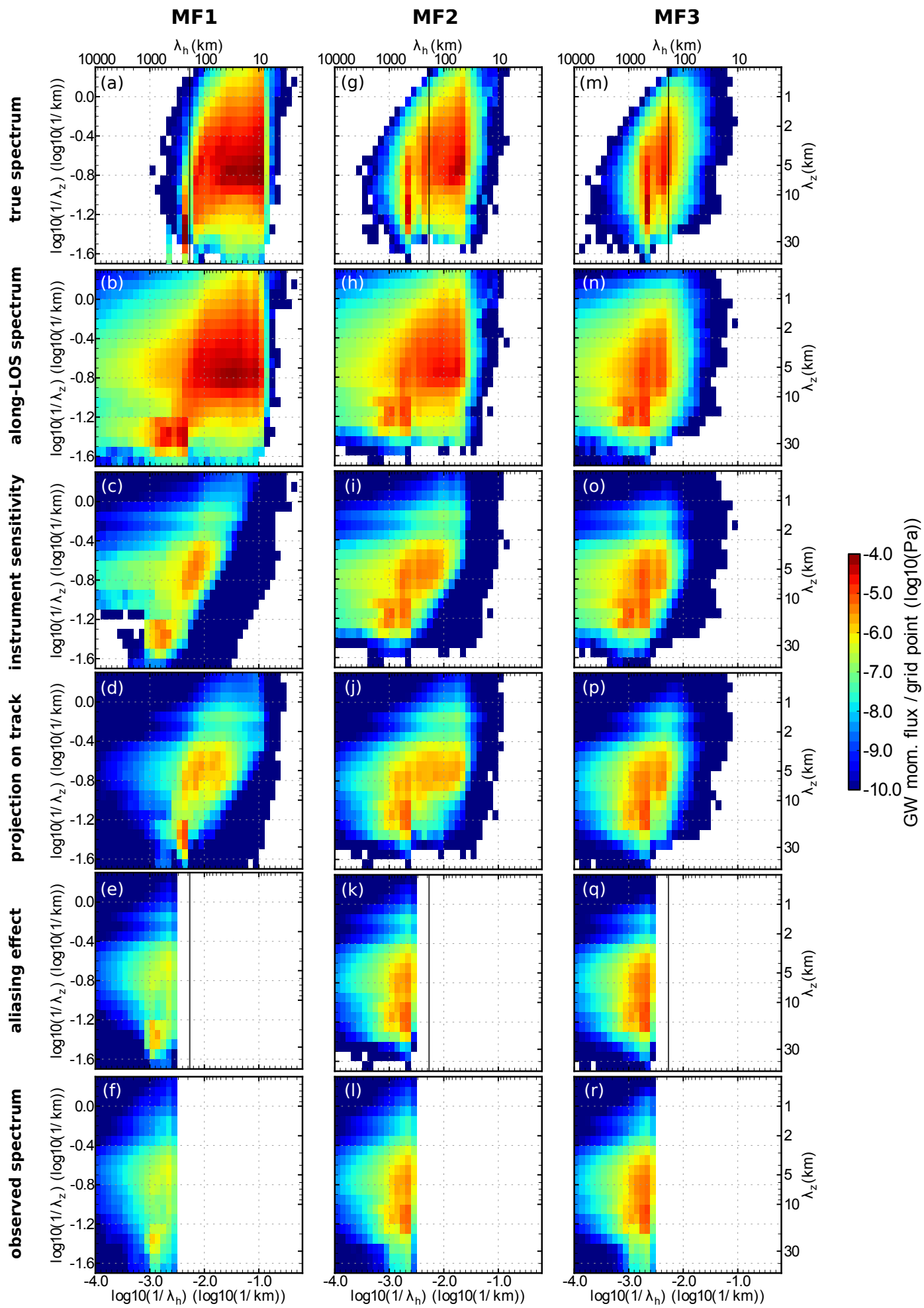

Figure 12. Application of the observational filter to MF1 (left column) MF2 (middle column), and MF3 (right column) for January 2008 with the observation geometry of SABER. Black vertical lines in the first and 5 th rows indicate $\lambda_{\mathrm{h}}=185 \mathrm{~km}$. 

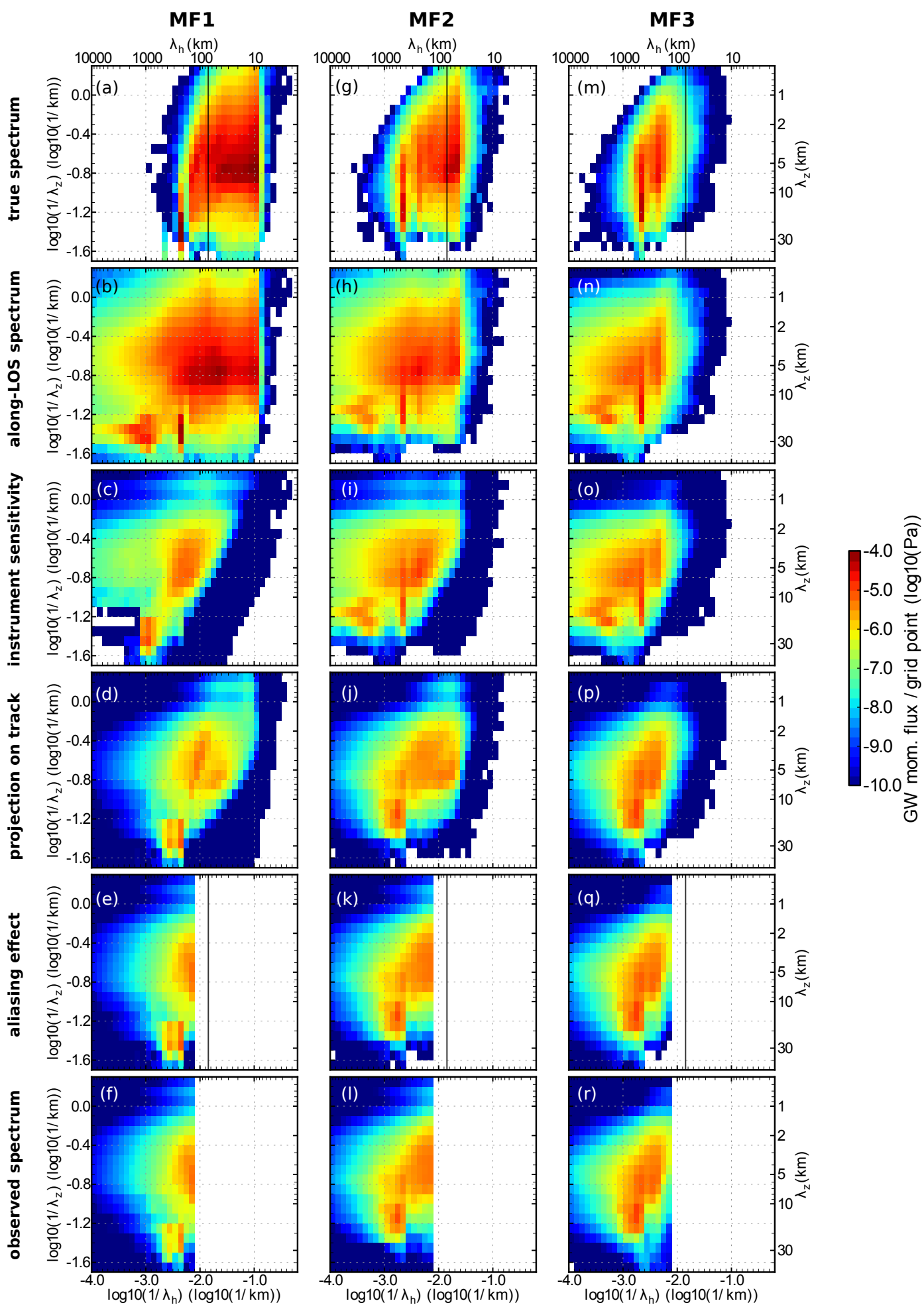

Figure 13. Application of the observational filter to MF1 (left column), MF2 (middle column) and MF3 (right column) for January 2008 with the observation geometry of HIRDLS. Black vertical lines in the first and 5 th rows indicate $\lambda_{\mathrm{h}}=70 \mathrm{~km}$. 

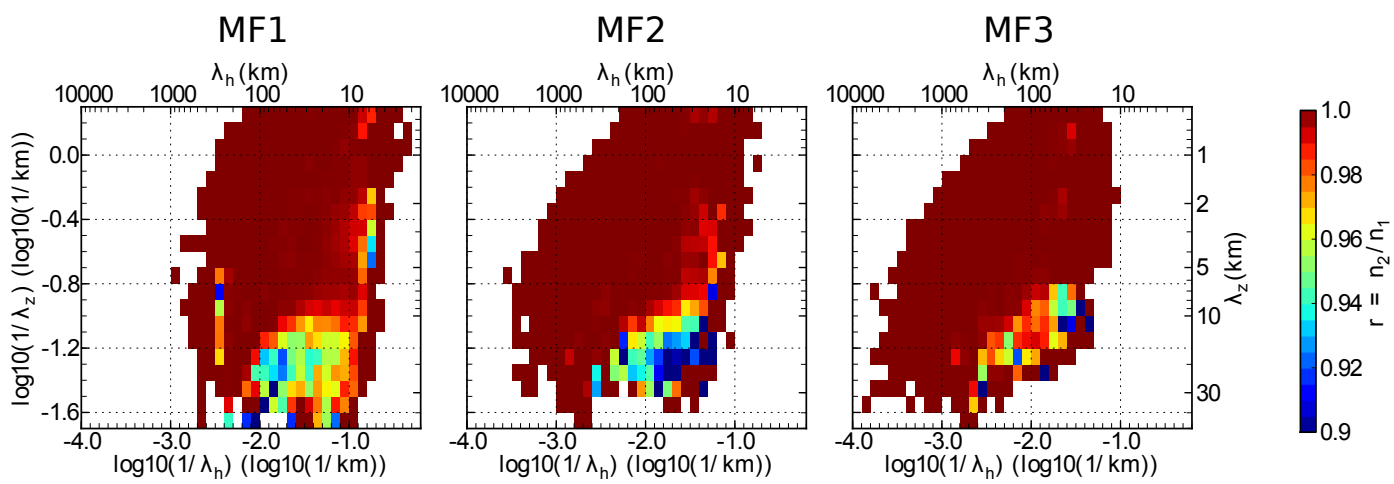

Figure 14. Variation of the number-of-wave-event spectrum after considering the difference between the observed vertical wavelengths for upward and downward scans. The variation is shown here for MF1 (left column), MF2 (middle column) and MF3 (right column) with the observation geometry of HIRDLS. For details, see text.

in the vertical direction down to $\lambda_{z}$ of about $2 \mathrm{~km}$, while for SABER this limit was about $4 \mathrm{~km}$. In the horizontal direction, since spectra of HIRDLS geometry spread more strongly with respect to $\lambda_{\mathrm{h}, \mathrm{LOS}}$, GWs appeared to be more sensitive to the instrument. Hence, the reduction of GWMF in the horizontal direction was also lower than for spectra based on SABER geometry.

This better conservation of GWMF for HIRDLS was also found in spectra of $F_{\text {track }}$ with respect to $\lambda_{\mathrm{h} \text {, track }}$ and $\lambda_{z}$ (cf. Fig. 13d, j, and p). The contribution of short verticalwavelength GWs to these spectra is more pronounced than in the case of SABER (Fig. 12d, j, and p).

Moreover, due to the shorter sampling distance, spectra in the case of HIRDLS were less influenced by aliasing than for SABER. Comparing aliasing-effect spectra of HIRDLS (Fig. 13e, k, and q) and SABER (Fig. 12e, k, and q), it is evident that for HIRDLS, a smaller part of the respective spectrum for MF1 was cut and for MF3 projected towards longer horizontal wavelengths (before the aliasing effect could take effect). The remaining part of each spectrum is therefore larger and more features are conserved.

In particular, the spectrum for MF3 and HIRDLS including the observational filter shown in Fig. 13q is the only one which has a well-resolved maximum that also decreases at short horizontal wavelength, similar to the observations of Ern and Preusse (2012). For this case (MF3), the spectral peak of the "true" spectrum is indeed captured by the observations.

Concerning the effect of " $\lambda_{z}$ restriction", only an insignificant variation was found in the number of wave events for SABER (not shown). In the case of HIRDLS, this variation was more pronounced, and the variation of the number-ofwave-event spectrum for HIRDLS is shown in Fig. 14. In this figure, the spectrum of the ratio $r=n_{2} / n_{1}$ is plotted with respect to the true horizontal and vertical wave numbers. Here, $n_{1}$ is the number of wave events in one bin before considering $\lambda_{z}$ restriction, $n_{2}$ is the number of wave events in the same bin after considering this restriction. Re- duced ratios were found in the lower right corner of the spectrum for all MF1, MF2 and MF3. This indicates that most of the filtered-out waves have short horizontal wavelength and long vertical wavelength. This can be explained as follows: when the horizontal wavelength is much longer than the vertical wavelength, the wave fronts are almost parallel to the horizon. In this case, the angle between the wave vector $\boldsymbol{k}$ and the normalized profile vector $\boldsymbol{p}$ is almost the same for upward scanning and downward scanning. Following Eqs. (18)-(20), the difference between observed vertical wavelengths in those two cases (upward and downward scan, respectively) is therefore insignificant. However, when horizontal wavelength and vertical wavelength are of the same order of magnitude, angles between $\boldsymbol{k}$ and $\boldsymbol{p}$ for upward and downward scanning are strongly different. This leads to a considerable difference in the observed vertical wavelengths. Figure 14 shows that even in the bins which were most affected, maximum profile loss was only about $10 \%$. On the other hand, profile loss in deriving data from HIRDLS observations using the method of Ern et al. (2011) was about $50 \%$ (Geller et al., 2013). This indicates that the $\lambda_{z}$ restriction step cannot be the major reason for the observed loss of about $50 \%$ of altitude profiles in real observations.

Figure 13f, 1, and $r$ shows "observed" spectra in the case of HIRDLS observation geometry. Again, in comparison with the aliasing-effect spectra, only minor changes were found and these changes were analogous to the case of SABER.

Overall, similar to the case of SABER, MF3 was least affected, while MF1 was most affected by aliasing. In particular for MF3, with observation geometry of HIRDLS, it was shown that almost all spectral features are preserved.

To conclude, for both cases of observation geometry (SABER and HIRDLS), all spectra (MF1, MF2 and MF3) shifted to the direction of longer horizontal as well as vertical wavelengths. A rather large part of each spectrum associated with short horizontal wavelengths was projected to longer horizontal wavelengths. The spectrum for MF3 has the longest spatial scale and was least influenced by the ob- 

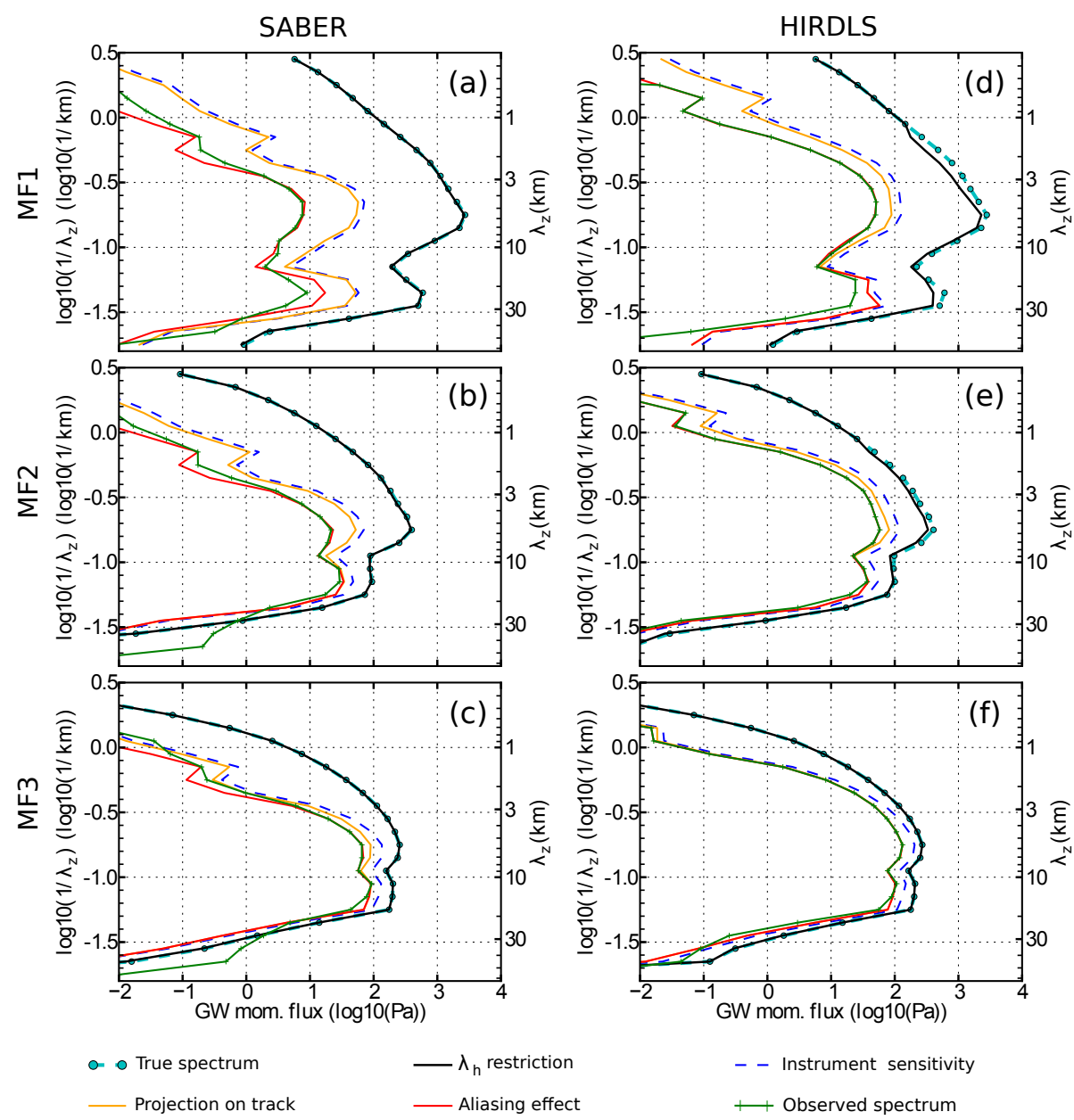

- - Instrument sensitivity

+ Observed spectrum

Figure 15. GWMF reduction during the observational filtering for (a, d) MF1, (b, e) MF2, and (c, f) MF3 with the observation geometry of SABER (left column) and HIRDLS (right column).

servational filter. In contrast, the spectrum for MF1 has the smallest spatial scale and was most influenced by the observational filter. The better sensitivity of HIRDLS helps to decrease the reduction of GWMF due to instrument sensitivity. In addition, HIRDLS's shorter sampling distance allows us to see a larger part of spectra after aliasing.

\subsection{Quantification of GWMF reduction}

As shown above, the magnitude of GWMF is decreased after applying filters mimicking $\lambda_{\mathrm{h}}$ restriction, instrument sensitivity, and aliasing. Moreover, the magnitude of GWMF also changes by calculating the observed vertical wavelength, the observed-vertical-wavelength restriction and additional correction. The changes during these last three steps, however, were minor, as we have seen from the spectra. In order to quantify the change in GWMF during the process of filtering, GWMF were integrated over all horizontal wave numbers and afterwards plotted against the vertical wave number in a base 10 logarithmic scale. The effects of the last three steps are discussed as one common step.

Figure 15 shows GWMF for SABER (left column) and HIRDLS (right column). The cyan dashed-dot line indicates GWMF of the true spectrum, the black solid line is GWMF after considering $\lambda_{\mathrm{h}}$ restriction, the blue dashed line presents GWMF after the instrument sensitivity has been considered, the orange line is GWMF after projecting on tangent-point track, the red line shows GWMF after the aliasing effect and the green line with crosses shows GWMF of the observed spectrum.

For both SABER and HIRDLS, the reduction due to the whole filtering process is largest for MF1 and smallest for MF3. It is indicated by the notable difference between GWMF of the true spectrum (cyan dashed-dot line) and the observed spectrum (green line with crosses). It is about 2.5 orders of magnitude for MF1 viewed by SABER (Fig. 15a) and about 2 orders of magnitude for MF1 viewed by HIRDLS (Fig. 15d). This difference is smaller in the case of MF2 (Fig. 15b and e) and is smallest in the case of MF3 
(Fig. 15c and f). For MF3, the difference is only about half an order of magnitude. This agrees well with the fact that the spectrum for MF1 is most influenced and the spectrum for MF3 is least influenced by the observational filter, as discussed above.

Moreover, for all spectra and for both observation geometries, it is clear that the instrument sensitivity is the factor that reduces GWMF the most. This reduction can be seen by comparing the black line and the dashed blue line. The difference between these two lines is the largest difference between two adjacent lines in all panels. Moreover, this reduction was strongest for MF1, decreasing from MF1 to MF3 due to the increase in spatial scales. Again, this finding is in agreement with the change in spectra described above in this section.

In addition, the reduction in the case of HIRDLS was weaker than in the case of SABER, which is explained by the better sensitivity of the HIRDLS instrument. For example, after considering the instrument sensitivity of HIRDLS, GWMF of MF1 and MF2 (Fig. 15d and e) was about 2.12.2 (in the unit of base 10 logarithmic scale), while for SABER, the value of GWMF dropped to about 1.8 (Fig. 15a and $b$ ). The contribution of short vertical-wavelength GWs from about 1 to about $3 \mathrm{~km}$ was also much larger in the case of HIRDLS than for SABER. For MF3 (Fig. 15c and f), the difference between these two observation geometries was lower than for MF1 and MF2; however, it is still recognizable even in the base 10 logarithmic scale.

The second strongest factor of GWMF reduction for SABER is aliasing, as can be seen by comparing the orange and the red lines, which are separated quite clearly from each other (except in the case of MF3). Again, the effect of aliasing decreases from MF1 to MF3 due to the increase in the spatial scales of the waves. Moreover, since the sampling distance of HIRDLS is shorter $(70 \mathrm{~km})$ than for SABER $(185 \mathrm{~km})$, less GWMF reduction by aliasing was found for HIRDLS.

The process of projecting the horizontal wavelength on the tangent-point track reduces GWMF less than instrument sensitivity and the aliasing effect in most cases; the exceptions are MF2 and MF3 for HIRDLS. Furthermore, the reduction by this factor was very similar for all spectra MF1, MF2 and MF3. This is due to the fact that the reduction is mainly induced by $|\cos (\psi-\gamma)|$, which does not depend on the spatial scale of the individual waves.

Minor redistribution of the spectra by the last three steps is shown by the difference between the red line and the green line with crosses. GWMF values at the spectral peak (at a vertical wavelength of about $30 \mathrm{~km}$ ) were reduced by the additional correction. This can be seen clearly in Fig. 15a and d.

The step of $\lambda_{\mathrm{h}}$ restriction affected GWMF least. In the base 10 logarithmic scale, GWMF of true spectrum (the cyan dashed-dot line) and GWMF after considering $\lambda_{\mathrm{h}}$ restriction (the black solid line) were nearly the same in almost all panels.
Table 1. Percentages of remaining GWMF at main steps during the observational filter.

\begin{tabular}{llrr}
\hline \multirow{2}{*}{ Spectrum } & Step & SABER & HIRDLS \\
\hline \multirow{2}{*}{ MF1 } & $\lambda_{\text {h }}$ restriction & 97.50 & 77.35 \\
& instrument sensitivity & 3.09 & 5.95 \\
& projection on track & 2.58 & 4.28 \\
& aliasing & 0.54 & 2.75 \\
& observed spectrum & 0.39 & 2.28 \\
\hline \multirow{2}{*}{ MF2 } & $\lambda_{\text {h restriction }}$ & 98.05 & 85.55 \\
& instrument sensitivity & 18.47 & 32.13 \\
& projection on track & 13.55 & 22.00 \\
& aliasing & 8.35 & 17.65 \\
& observed spectrum & 7.65 & 17.13 \\
\hline \multirow{2}{*}{ MF3 } & $\lambda_{\text {h } \text { restriction }}$ & 99.72 & 99.79 \\
& instrument sensitivity & 46.32 & 67.55 \\
& projection on track & 31.91 & 44.86 \\
& aliasing & 27.21 & 44.80 \\
& observed spectrum & 25.62 & 43.52 \\
\hline
\end{tabular}

In addition, cyan dashed-dot lines in Fig. 15 (true spectra) show that GWMF given by MF1 is the largest with a peak at about 3.5 (in the base 10 logarithmic scale). For MF2, this value is about 2.7 and for MF3 it is only about 2.4. The relative importance of these different spectra (MF1, MF2, MF3) in the whole GWMF spectrum is, however, still unknown and may be adjusted (e.g., by intermittency or efficiency factors), as the relative importance of various convective process in exciting GWs is still badly constrained.

More details about the reduction in GWMF during the observational filter are presented in Table 1. Here, the total GWMF of the true spectrum is $100 \%$. The percentages of the remaining GWMF in other steps of the observational filter (instrument sensitivity, projection on track, aliasing effect and observed spectrum) are shown for all spectra.

\section{Conclusions}

Prior publications have revealed the importance of the observational filter. Observational filters for different measurement techniques have been studied with a special focus on instrument visibility (e.g., Alexander, 1998; Preusse et al., 2000) or careful consideration of observation geometry (e.g., $\mathrm{Wu}$ and Eckermann, 2008). In this study, for the first time, a comprehensive observational filter for infrared limb sounders with a high level of accuracy, which takes into account the visibility of waves to an infrared limb sounder as well as a sophisticated representation of the observation geometry, was developed.

The comprehensive observational filter contains four main processes: visibility filter, projection of the wavelength on the tangent-point track, aliasing effect and the calculation of the vertical observed wavelength. The first process com- 
prises the following elements: the determination of the wavelength along the LOS, restriction of horizontal wavelength, application of the approximate sensitivity function (radiative transfer). The second process includes the determination of the along-track wavelength and the calculation of the corresponding GWMF. The third process calculates the projection of waves towards much longer wavelengths by aliasing and the associated reduction of GWMF. The last step calculates the vertical wavelength which would be observed by the instrument and the corresponding GWMF. An additional correction is also applied in this last process.

The observation geometries of SABER and HIRDLS instruments were considered in our study. The results show that the most important processes, which have significant influences on the spectrum, are as follows: visibility filter (for both SABER and HIRDLS observation geometries), aliasing for SABER, and projection on tangent-point track for HIRDLS.

We found that the vertical wavelength distribution was mainly affected by the "visibility filter" process, which relates to the radiative transfer and retrieval. This process reduced the short vertical-wavelength GWs, but did not largely change the shape of the vertical-wavelength spectrum. This is shown in Fig. 15. In this figure, all panels other than Fig. 15a show largely the same vertical wavelength distribution and in particular the peak at the same vertical wavelength as the original spectrum. For the horizontal structure, depending on the horizontal scale of the original spectrum, the observational filter can have stronger or weaker effects. For the original spectrum containing a short horizontal scale, in addition to the significant influence of the visibility filter, the spectrum was projected onto a longer horizontal wavelength interval which originally was not populated. In this case, a strong contribution to the spectrum was found until the Nyquist wavelength. In other words, a pronounced spectral peak, which stands out from other parts of the spectrum, was not generated. GWMF for this case (MF1) was largely reduced, possibly making such spectral contributions difficult to observe by infrared limb instruments. In the case of the long-horizontal-scale original spectrum, a pronounced peak was found. This finding suggests that a pronounced spectral peak is an indication of longer horizontal wavelengths in the original distribution.

We also found that during the filtering procedure, GWMF values of the spectrum containing very short horizontal wavelengths were reduced considerably. Moreover, due to the measurement geometry, altitude profiles are oblique, which results in a slight shift of the vertical wavelength. Simulating this effect, we find that it does not affect the evaluation of profile pairs which need to match closely in their vertical wavelength (Ern et al., 2011). For average spectra the overall effect is a negligible shift in the vertical wavelength distribution. In the current work, calculations were averaged for ascending and descending orbits because no significant differences between two of them were found (not shown).
However, this depends on the particular observation geometry of each instrument. For another instrument, these differences might be significant and may have to be taken into account. In addition, error caused by the instrument noise, as discussed below in Appendix B, is negligible and therefore is not considered in this observational filter.

The comprehensive observational filter is a powerful tool for comparing GW modeling with observations. This can be applied, as in our case, to the modeling of individual monochromatic waves by a single-wave GW model. However, also numerical model data can be spatially and spectrally decomposed. For instance, Preusse et al. (2014) used monochromatic fits in small volumes for comparing ECMWF data to observations. In their work, this observational filter was applied in order to increase the significance of the observation. Our main interest is the meaningful comparison between global observations and global GW modeling with uncertainties smaller than those uncertainties assumed for global GW observations alone (Ern et al., 2004; Geller et al., 2013). This shall result in improved understanding of the distributions of GWs in the real world and, hopefully, in realistic representations of GWs in GCMs employed for weather prediction and climate projection.

The example of the three parameter sets of convective GWs may be taken as a first example how such constraints of global GW modeling may work. In addition to GWMF it is also possible to obtain estimates of the so-called "residual drag" from assimilated data (Alexander and Rosenlof, 2003; Pulido and Thuburn, 2006; Ern et al., 2014). Observed GWMF and the drag exerted by GWs can be used together to constrain e.g., a GW parameterization scheme. For instance, it is believed that convective GWs are a main driver of the QBO. There is an evidence that IR limb sounders see roughly half of that GW driving (Ern et al., 2014). However, since limb sounders see only $1 \%$ of the GWMF from MF1 (i.e., limb sounders almost do not see them at all), MF1 may thus contribute a maximum of $50 \%$ to the QBO driving. If one first obtains a good match to the waves visible, the constraints for MF1 become sharper. However, in order to infer an upper limit to the GWMF from MF1 one has first to calculate for each of the spectral components from MF1 to MF3 the drag they potentially exert and one has to solve the momentum balance in the QBO. Such a quantitative assessment involving also ranges of uncertainties is far beyond the aim of this technical paper, but the way forward is clear.

The MF1 peak and MF2 peak discussed in this study follow a consistent concept of subgrid convection parameterization and subgrid GW parameterization. In the case of MF3 the spatial scale assumed for the convection may exceed the scale of a model grid, while the assumption of the convection parameterization is based on a subgrid process. Though this is a conceptual inconsistency, we may still technically need to parameterize such waves; because the dynamical feedback between the convection parameterization and the GCM is too 
weak, they may not be excited in terms of resolved GWs (Preusse et al., 2014).

This observational filter is also helpful for interpreting the real observed spectra, since the horizontal and vertical structures of the original spectral distributions might be better predicted. 


\section{Appendix A: Applicability to other types of instruments}

In the main body of this paper, we have discussed the observational filter for GWMF from spaceborne observations and focused on the case of instruments measuring optically thin emissions in limb scanning geometry. The measurement method determines the visibility filter as well as the observation geometry. Other kinds of instruments require different observational filters. In this section we will describe whether and how the general approach outlined here may be adapted to other techniques. We will start this by reconsidering some general limitations.

The direct inference of GWMF from wind perturbations requires to measure instantaneously all three components of the wind with an accuracy which cannot be reached from space with any technique existing or under development. Instead, estimates of GWMF are based on the polarization relations and require to determine the horizontal and vertical wavelength in addition to the temperature amplitude (Ern et al., 2004). In general, the phase of a gravity wave changes both in space and time and, analyzing the wavelengths and periods of a GW, this information must not be mixed (e.g., de la Torre and Alexander, 1995; de la Torre et al., 1999; Eckermann et al., 2006). However, considering a snapshot, we may focus on the spatial variations only and disregard temporal evolutions. As shown by Alexander et al. (2010), the shortest intrinsic periods visible to infrared limb sounding are $\sim 1 \mathrm{~h}$. Low Earth orbit (LEO) satellites have a velocity of $\sim 8 \mathrm{~km} \mathrm{~s}^{-1}$; i.e., a typical $\mathrm{GW}$ wavelength of $500 \mathrm{~km}$ or less is covered in less than $1 \mathrm{~min}$. It is therefore safe to assume measurements from one orbit-segment to be instantaneous. In contrast, the duration of an orbit is $\sim 1.5 \mathrm{~h}$. Although at the turning latitudes of the orbit, subsequent orbits may be sufficiently close for considering the same GW event, the phase of this wave likely has changed in the $1.5 \mathrm{~h}$ which passed between these observations. For emission sounding with a single instrument, it is therefore not promising to combine the observations of subsequent orbits, nor is it promising to combine two instruments on different platforms. Of course, insight can be gained in case studies by revisiting the same region (e.g., Preusse et al., 2002; Eckermann et al., 2006, 2007), but one should not combine the phase information to infer wavelengths.

\section{A1 Potential future limb imager}

Gravity wave information has been retrieved from a number of infrared limb sounders, that is CRISTA (e.g., Preusse et al., 2002; Ern et al., 2006), Cryogenic Limb Array Etalon Spectrometer (CLAES) (Preusse and Ern, 2005), SABER (Preusse et al., 2009a; Ern et al., 2011) and HIRDLS (e.g., Alexander et al., 2008; Wright et al., 2010; Ern et al., 2011). Only CRISTA uses multiple viewing directions, but the observation tracks are separated by $600 \mathrm{~km}$ and thus too far apart for common analysis of the same GW events. In ad- dition, all these instruments had to assume spherical symmetry for the retrieval, and they cannot observe wavelengths which are shorter than the Nyquist wavelength of their sampling. Thus, the complex visibility filter, the projection of the wave to the measurement track and aliasing are inevitable for these instruments. They could, however, be remedied, if an instrument were designed for the purpose of measuring GWs (Riese et al., 2005; Preusse et al., 2009b). Viewing backward and sampling sufficiently frequently, 2-D tomographic retrievals can be employed, which allow to reconstruct the true amplitude in that part of the spectrum generally visible to limb sounders (Ungermann et al., 2010). This largely simplifies the visibility filter and strongly reduces its effect. Using 2-D imaging, also across-track information would be achieved, which would allow for reconstruction of the 3-D wave vector. Accordingly, the projection to the tangent-point track becomes obsolete. Finally, oblique-profile effects are removed in the retrieval.

\section{A2 Microwave limb sounder}

In contrast to infrared limb sounders, for the microwave limb sounder (MLS) both on Upper Atmosphere Research Satellite (UARS) (Wu and Waters, 1996a, b) and Earth Observing System (EOS)-Aura (Wu and Eckermann, 2008) saturated radiances were utilized for GW studies. In this case the radiance does not stem from the tangent point, but from a part of the limb ray which is higher in altitude and closer to the instrument. The altitude associated with these observations is determined by the wavelength of the microwave radiation chosen for the analysis, and the sensitive volume is oriented oblique in the atmosphere. Accordingly, this geometry is called sub-limb (Wu et al., 2006). Sub-limb observations have a strong bias of waves (intrinsically) propagating towards the instrument (Jiang et al., 2004) and are most sensitive for waves at the edge of the visibility range of limb sounders (McLandress et al., 2000; Preusse et al., 2008). Due to the lack of vertical wavelength information, only in a single instance GWMF from MLS was published (Jiang et al., 2006). For the comparison of modeled and measured radiance variances, the observational filter described here may be adapted. The equations for the visibility filter can be taken from McLandress et al. (2000). In the case of MLS the large-scale structures of the atmosphere (e.g., planetary waves) are removed by along-track high-pass filtering. This is an essential part of the observational filter. For UARS MLS, which views $90^{\circ}$ to the flight direction, along-LOS projection for the visibility filter and along-track projection for the background removal need to be considered separately (Jiang et al., 2004). For AURA-MLS which views forward, both steps may be combined in one observational filter. 


\section{A3 GPS-RO}

So far we have considered techniques where measurements were taken by a single instrument and the spatiotemporal collocation was reached by considering subsequent measurements on one orbit segment. In this case any given wave can be viewed only by a very limited number of geometries, i.e., ascending/descending orbit nodes and, in the case of SABER, southward/northward looking viewing mode. The situation is very different for radio occultations between dedicated receiver satellites and transmitter satellites of the global positioning system (GPS-RO). For the COSMIC mission this involved 12 transmitter satellites and 6 receiver satellites resulting in $\sim 2000$ GPS-RO profiles per day distributed quasi-randomly over the globe. Using maximum miss distances and miss times such as $15^{\circ}$ and $2 \mathrm{~h}$, groups of three profiles may be identified, from which to infer the 3-D wave vector (Wang and Alexander, 2010; Faber et al., 2013). This results in triples with an average distance of $1000 \mathrm{~km}$ and a sufficient number of events to generate meaningful seasonal-average maps. This different procedure has a number of consequences for the observational filter. First, the wave is viewed from different directions. This may not only lead to different amplitude degradations for the individual profiles, but also introduce different phase shifts in each of the profiles of the triple (Belloul and Hauchecorne, 1997; Preusse et al., 2002). Second, in order to gain the best estimate of the 3-D wave vector a complicated phasedewrapping is required (Faber et al., 2013). Because of these two points, it is likely best to estimate observational filter effects by performing the phase dewrapping for three given profile locations from simulated phases, which are calculated by applying individual LOS projection and visibility filtering including phase shifts. Finally, the general concept needs to be changed. In the case of the emission sounders, for each wave the latitude position determines how this wave is viewed. In the case of GPS, a certain region may be viewed by completely different combinations of viewing geometries. That could, for instance, be solved by a stochastic approach.

\section{A4 Nadir sounding}

There is a number of studies utilizing nadir sounding of thermal emissions in the infrared and microwave spectral region. This technique has the advantage of resolving the horizontal wave structure. However, GWMF was only deduced in case studies, for example for the AIRS instrument (Alexander and Teitelbaum, 2011). Nadir sounding satellites have, in principle, a more simple geometry. The horizontal resolution depends mainly on the footprint size and sampling, the vertical resolution is given by the radiative transfer. For the outer track the geometry approaches sub-limb and the observational filter becomes more complicated (Eckermann et al., 2007). The latter allows also for deducing directional propagation preferences from AIRS radiances (Gong et al., 2012).
Finally, the actual observational filter may depend as much on the analysis technique as on the instrument itself.

\section{A5 Summary}

To sum up, the observational filter described in the current paper can be adapted to other measurement techniques. In particular, for the potential future limb imager, the visibility filter will be more simple. Projection of the wave on the measurement track, aliasing effect and oblique-profile effect are not further needed. In the case of MLS, for radiance variances, the observational filter can also be adapted using equations from McLandress et al. (2000) for the visibility filter, and considering the large-scale structure removal by along-track high-pass filtering. However, much more effort is needed to adapt the current observational filter to GPS-RO; in contrast to emission measurements, the observations geometry varies with each individual sounding even for a given latitude. True nadir sounding has a simpler observational filter than infrared limb and can be treated accordingly. Finally, it is important to mention that the infrared limb sounding technique can cover a large part of the GW spectrum. A comprehensive observational filter for this technique therefore is essential for quantitatively confining resolved and parameterized GWs in global models.

\section{Appendix B: Background removal and noise}

In this paper we have developed the observational filter for infrared limb sounders. The observational filter was defined in a deterministic way, and the different involved steps are inevitable. For a given wave and a given instrument (including orbit direction), all effects described here will apply only in the way as they are described here. These effects do not depend on the specific method used for the interpretation of the data, for instance, which kind of spectral analysis is used for the vertical profiles (e.g., whether to use MEM/HA, Preusse et al., 2002; S-transform, Alexander et al., 2008, or multi-component S-transform, Wright and Gille, 2013). This independence of these effects works well in the case of infrared limb sounders, since the background removal does not strongly affect the visible wavelengths and since the instrument noise level is low. This separability is not given e.g., in the case of MLS, where the background removal significantly influences the visible wavelengths (cf. MLS in Appendix A). Of course background removal and noise still may influence the measured GWMF distributions, but it is much more straightforward to take them into account in the error estimates of the measured distribution. We will discuss both effects briefly in this section.

\section{B1 Background removal}

In the case of infrared limb sounders the background is usually removed by determining planetary waves up to wave 
number 6 and subtracting these waves from the individual measurements. Though the basic approach is the same, different techniques have been applied including Kalman filter (Fetzer and Gille, 1994), Kalman filter and additional split into ascending and descending orbits for tidal removal (Preusse et al., 2001), S-transform (Alexander et al., 2008) and spatiotemporal decomposition (Ern et al., 2011, 2013). Though the wave number 6 is technically driven by the orbit geometry of LEOs, which allows for the determination of planetary waves up to a maximum of 7 (Salby, 1982), it turns out to be a rather good choice at least for the stratosphere and mesosphere. The main contributions of planetary scale waves in terms of variances are mainly contained in zonal wave numbers up to 4 (e.g., Ern et al., 2008; Ern and Preusse, 2009). This means that planetary waves can be completely removed. Gravity waves have much shorter wavelengths than zonal wave number 6 and are therefore not removed. The latter was shown, for instance, by Preusse et al. (2006) who find that the horizontal wavelength distribution of measured GWs follows largely a fixed ratio between intrinsic frequency of GWs and Coriolis parameter $\hat{\omega} / f$ than following the wave number limit which would have been implied by the background removal. Though the background removal thus does not influence the observational filter, the determination of the planetary scale waves is an error source. If these waves are not captured in full, GW variance will be overestimated, if part of the GW structure is erroneously projected into planetary scale waves, $\mathrm{GW}$ variance will be underestimated.

\section{B2 Noise}

The noise level of infrared limb sounders is typically a fraction of $1 \mathrm{~K}$ in the stratosphere and typically $1 \mathrm{~K}$ in the upper mesosphere. Noise levels for HIRDLS and SABER as well as the references where to find them are given in Ern et al. (2014). This compares to typical GW amplitudes of a few K in the stratosphere and more than $10 \mathrm{~K}$ in the upper mesosphere. Noise for the leading spectral components employed for GWMF estimation is further reduced by using a number of points in the spectral analysis of the vertical profiles. In regions of prominent sources and favorable propagation conditions, the influence of noise is hence at least an order of magnitude below the typical size of GW variance and GWMF. The case may be different in the summer high-latitude lower stratosphere where the wind reversal between tropospheric westerlies and stratospheric easterlies largely prevents GWs from entering the stratosphere (cf. e.g., Kalisch et al., 2014, and references therein). In this region, noise may indeed have a larger influence on the determined level of GWMF and this region could be used for a check of the noise-induced background level of GW variance and GWMF.

For the technique applied in our own research, one may perform kind of a plausibility check. For the evaluation of GWMF we use only the major spectral component. In regions where GWs are prominent, the influence of noise on this component is marginal (see paragraph above). We now can compare the total variance determined directly from the temperature residuals after background removal (P1), the total variance of GWs from the major spectral component as analyzed for single profiles (P2), and the total variance of GWs from profile pairs where the vertical wavelength of the two single profiles reasonably well agree (P3). Since the vertical wavelength agrees in profile pairs, one may argue that one chiefly has captured true GW events, whereas in the single profiles there could be a higher fraction of results dominated by noise. However, the variance values for single profiles (P2) and profile pairs (P3) agree very well (Ern et al., 2014; Geller et al., 2013). This is a plausibility check that after the spectral analysis, the contribution of noise is low. On the other hand the major spectral component captures about $70 \%$ of the initial variance (Ern et al., 2014). This means that we likely have also captured the main part of the GWMF. Since at a certain location likely more than one GW is found quite frequently, also the remaining part of the variance is probably dominated by GWs. This, in turn, indicates that even in the direct variance estimate, the contribution of noise is quite small.

The observational filter of infrared limb sounders as described here is deterministic and independent of the individual evaluation method. The removal of the background and instrument noise will cause different GWMF errors depending on the chosen method. Noise and background removal therefore rather belong to the error of the distribution than to the application of the observational filter and may be estimated e.g., by simulated data in a Monte Carlo simulation. This is, however, not the topic of the current paper.

\section{Appendix C: Dependences of $\beta$ and $\gamma$ on latitude}

Figure $\mathrm{C} 1$ shows variations of $\beta$ and $\gamma$ against latitude. In particular, Fig. $\mathrm{Cla}$ and $\mathrm{b}$ present the variances for the northward-viewing mode and Fig. $\mathrm{C} 1 \mathrm{c}$ and $\mathrm{d}$ for the southward-viewing mode of SABER. For HIRDLS, the dependences of $\beta$ and $\gamma$ on latitude are shown in Fig. Cle and $\mathrm{f}$. For all panels in this figure, ascending orbit is presented in the left column and descending orbit is in the right column. 

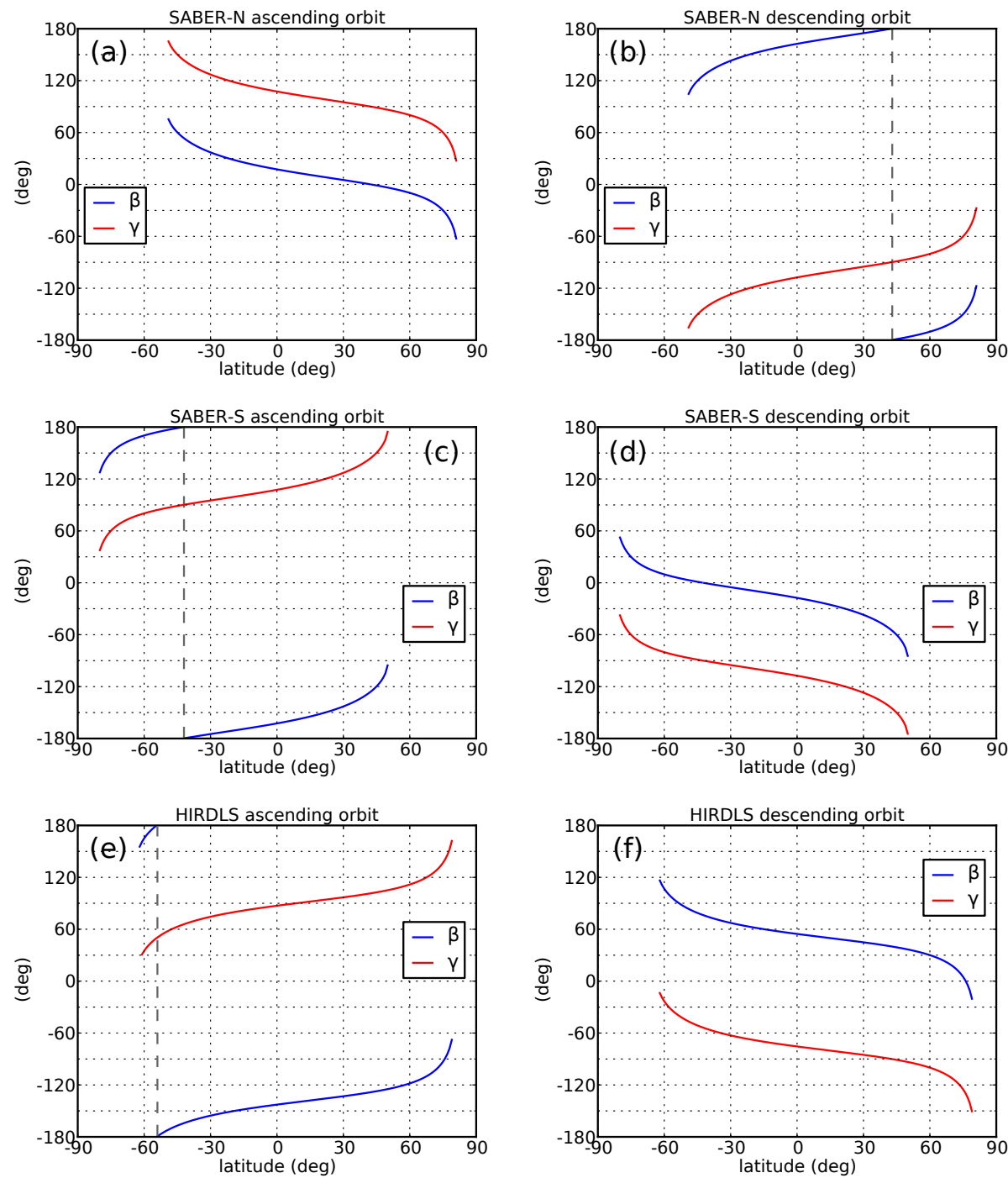

Figure C1. Dependences of $\beta$ and $\gamma$ on latitude for different orbit directions of (a-d) SABER and (e, f) HIRDLS. For details, see text. 
Acknowledgements. MERRA data and information on the HIRDLS instrument were provided by NASA. Information on the SABER instrument was provided by GATS Inc.

The article processing charges for this open-access publication have been covered by a Research Centre of the Helmholtz Association.

Edited by: M. Rapp

\section{References}

Alexander, M. J.: Interpretations of observed climatological patterns in stratospheric gravity wave variance, J. Geophys. Res., 103, 8627-8640, 1998.

Alexander, M. J. and Ortland, D. A.: Equatorial waves in High Resolution Dynamics Limb Sounder (HIRDLS) data, J. Geophys. Res., 115, D24111, doi:10.1029/2010JD014782, 2010.

Alexander, M. J. and Rosenlof, K. H.: Gravity-wave forcing in the stratosphere: Observational constraints from the Upper Atmosphere Research Satellite and implications for parameterization in global models, J. Geophys. Res., 108, 4597, doi:10.1029/2003JD003373, 2003.

Alexander, M. J. and Teitelbaum, H.: Three-dimensional properties of Andes mountain waves observed by satellite: A case study, J. Geophys. Res.-Atmos., 116, D23110, doi:10.1029/2011JD016151, 2011.

Alexander, M. J., Gille, J., Cavanaugh, C., Coffey, M., Craig, C., Dean, V., Eden, T., Francis, G., Halvorson, C., Hannigan, J., Khosravi, R., Kinnison, D., Lee, H., Massie, S., and Nardi, B.: Global estimates of gravity wave momentum flux from High Resolution Dynamics Limb Sounder (HIRDLS) observations, J. Geophys. Res., 113, D15S18, doi:10.1029/2007JD008807, 2008.

Alexander, M. J., Geller, M., McLandress, C., Polavarapu, S., Preusse, P., Sassi, F., Sato, K., Eckermann, S. D., Ern, M., Hertzog, A., Kawatani, Y., Pulido, M., Shaw, T., Sigmond, M., Vincent, R., and Watanabe, S.: Recent developments in gravity wave effects in climate models, and the global distribution of gravity wave momentum flux from observations and models, Q. J. Roy. Meteorol. Soc., 136, 1103-1124, doi:10.1002/qj.637, 2010.

Allen, S. J. and Vincent, R. A.: Gravity wave activity in the lower atmosphere: Seasonal and latitudinal variations, J. Geophys. Res., 100, 1327-1350, 1995.

Bailey, L. P. and Gille, J. C.: Inversion of limb radiance measurements: an operational algorithm, J. Geophys. Res., 91, 27572774, 1986.

Belloul, M. B. and Hauchecorne, A.: Effect of periodic horizontal gradients on the retrieval of atmospheric profiles from occultation measurements, Radio Science, 32, 469-478, 1997.

Björn, L.: The cold summer mesopause, Adv. Space Res., 4, 145151, doi:10.1016/0273-1177(84)90277-1, 1984.

Butchart, N., Cionni, I., Eyring, V., Shepherd, T. G., Waugh, D. W., Akiyoshi, H., Austin, J., Brühl, C., Chipperfield, M. P., Cordero, E., Dameris, M., Deckert, R., Dhomse, S., Frith, S. M., Garcia, R. R., Gettelman, A., Giorgetta, M. A., Kinnison, D. E., Li, F., Mancini, E., McLandress, C., Pawson, S., Pitari, G., Plummer, D. A., Rozanov, E., Sassi, F., Scinocca, J. F., Shibata, K., Steil, B., and Tian, W.: Chemistry- climate model simulations of twenty-first century stratospheric climate and circulation changes, J. Climate, 23, 5349-5374, doi:10.1175/2010JCLI3404.1, 2010.

Choi, H.-J., Chun, H.-Y., and Song, I.-S.: Gravity wave temperature variance calculated using the ray-based spectral parameterization of convective gravity waves and its comparison with Microwave Limb Sounder observations, J. Geophys. Res., doi:10.1029/2008JD011330, 2009.

Choi, H.-J., Chun, H.-Y., Gong, J., and Wu, D. L.: Comparison of gravity wave temperature variances from ray-based spectral parameterization of convective gravity wave drag with AIRS observations, J. Geophys. Res., 117, D05115, doi:10.1029/2011JD016900, 2012.

de la Torre, A. and Alexander, P.: The interpretation of wavelengths and periods as measured from atmospheric balloons, J. Appl. Meteorol., 34, 2747-2754, doi:10.1175/15200450(1995)034<2747:TIOWAP>2.0.CO;2, 1995.

de la Torre, A., Alexander, P., and Giraldez, A.: The kinetic to potential energy ratio and spectral separability from high-resolution balloon soundings neat the Andes mountains, Geophys. Res. Lett., 26, 1413-1416, doi:10.1029/1999GL900265, 1999.

Dunkerton, T. J.: The role of gravity waves in the quasi-biennial oscillation, J. Geophys. Res., 102, 26053-26076, 1997.

Eckermann, S. D. and Marks, C. J.: GROGRAT: a New Model of the Global propagation and Dissipation of Atmospheric Gravity Waves, Adv. Space Res., 20, 1253-1256, 1997.

Eckermann, S. D., Hirota, I., and Hocking, W. K.: Gravity wave and equatorial wave morphology of the stratosphere derived from long-term rocket soundings, Q. J. Roy. Meteorol. Soc., 121, 149186, 1995.

Eckermann, S. D., Wu, D. L., Doyle, J. D., Burris, J. F., McGee, T. J., Hostetler, C. A., Coy, L., Lawrence, B. N., Stephens, A., McCormack, J. P., and Hogan, T. F.: Imaging gravity waves in lower stratospheric AMSU-A radiances, Part 2: Validation case study, Atmos. Chem. Phys., 6, 3343-3362, doi:10.5194/acp-63343-2006, 2006.

Eckermann, S. D., Ma, J., Wu, D. L., and Broutman, D.: A threedimensional mountain wave imaged in satellite radiance throughout the stratosphere: Evidence of the effects of directional wind shear, Q. J. Roy. Meteorol. Soc., 133, 1959-1975, 2007.

Ern, M. and Preusse, P.: Quantification of the contribution of equatorial Kelvin waves to the QBO wind reversal in the stratosphere, Geophys. Res. Lett., 36, doi:10.1029/2009GL040493, 2009.

Ern, M. and Preusse, P.: Gravity wave momentum flux spectra observed from satellite in the summertime subtropics: Implications for global modeling, Geophys. Res. Lett., 39, L15810, doi:10.1029/2012GL052659, 2012.

Ern, M., Preusse, P., Alexander, M. J., and Warner, C. D.: Absolute values of gravity wave momentum flux derived from satellite data, J. Geophys. Res., 109, D20103, doi:10.1029/2004JD004752, 2004.

Ern, M., Preusse, P., and Warner, C. D.: A comparison between CRISTA satellite data and Warner and McIntyre gravity wave parameterization scheme: horizontal and vertical wavelength filtering of gravity wave momentum flux, Adv. Space Res., 35, 20172023, doi:10.1016/j.asr.2005.04.109, 2005.

Ern, M., Preusse, P., and Warner, C. D.: Some experimental constraints for spectral parameters used in the Warner and McIntyre 
gravity wave parameterization scheme, Atmos. Chem. Phys., 6, 4361-4381, doi:10.5194/acp-6-4361-2006, 2006.

Ern, M., Preusse, P., Krebsbach, M., Mlynczak, M. G., and Russell III, J. M.: Equatorial wave analysis from SABER and ECMWF temperatures, Atmos. Chem. Phys., 8, 845-869, doi:10.5194/acp-8-845-2008, 2008.

Ern, M., Preusse, P., Gille, J. C., Hepplewhite, C. L., Mlynczak, M. G., Russell III, J. M., and Riese, M.: Implications for atmospheric dynamics derived from global observations of gravity wave momentum flux in stratosphere and mesosphere, J. Geophys. Res., 116, D19107, doi:10.1029/2011JD015821, 2011.

Ern, M., Preusse, P., Kalisch, S., Kaufmann, M., and Riese, M.: Role of gravity waves in the forcing of quasi two-day waves in the mesosphere: An observational study, J. Geophys. Res.-Atmos., 118, 3467-3485, doi:10.1029/2012JD018208, 2013.

Ern, M., Ploeger, F., Preusse, P., Gille, J. C., Gray, L. J., Kalisch, S., Mlynczak, M. G., Russell, J. M., and Riese, M.: Interaction of gravity waves with the QBO: A satellite perspective, J. Geophys. Res.-Atmos., 119, 2329-2355, doi:10.1002/2013JD020731, 2014.

Evan, S., Alexander, M. J., and Dudhia, J.: WRF simulations of convectively generated gravity waves in opposite QBO phases, J. Geophys. Res., 117, doi:10.1029/2011JD017302, 2012.

Faber, A., Llamedo, P., Schmidt, T., de la Torre, A., and Wickert, J.: On the determination of gravity wave momentum flux from GPS radio occultation data, Atmos. Meas. Tech., 6, 3169-3180, doi:10.5194/amt-6-3169-2013, 2013.

Fetzer, E. J. and Gille, J. C.: Gravity wave variances in LIMS temperatures, I, variability and comparison with background winds, J. Atmos. Sci., 51, 2461-2483, 1994.

Fritts, D. and Alexander, M.: Gravity wave dynamics and effects in the middle atmosphere, Rev. Geophys., 41, 1003, doi:10.1029/2001RG000106, 2003.

Garcia, R. R. and Randel, W. J.: Acceleration of the Brewer-Dobson circulation due to increases in greenhouse gases, J. Atmos. Sci., 65, 2731-2739, doi:10.1175/2008JAS2712.1, 2008.

Geller, M. A., Alexander, M. J., Love, P. T., Bacmeister, J., Ern, M., Hertzog, A., Manzini, E., Preusse, P., Sato, K., Scaife, A. A., and Zhou, T.: A comparison between gravity wave momentum fluxes in observations and climate models, J. Climate, 26, 6383-6405, doi:10.1175/JCLI-D-12-00545.1, 2013.

Gille, J. C., Barnett, J. J., Whitney, J. G., Dials, M. A., Woodard, D., Rudolf, W. P., Lambert, A., and Mankin, W.: The HighResolution Dynamics Limb Sounder (HIRDLS) experiment on AURA, Proc. SPIE, 5152, 161-171, 2003.

Gille, J. C., Barnett, J., Arter, P., Barker, M., Bernath, P., Boone, C., Cavanaugh, C., Chow, J., Coffey, M., Craft, J., Craig, C., Dials, M., Dean, V., Eden, T., Edwards, D. P., Francis, G., Halvorson, C., Harvey, L., Hepplewhite, C., Khosravi, R., Kinnison, D., Krinsky, C., Lambert, A., Lee, H., Lyjak, L., Loh, J., Mankin, W., Massie, S., McInerney, J., Moorhouse, J., Nardi, B., Packman, D., Randall, C., Reburn, J., Rudolf, W., Schwartz, M., Serafin, J., Stone, K., Torpy, B., Walker, K., Waterfall, A., Watkins, R., Whitney, J., Woodard, D., and Young, G.: High Resolution Dynamics Limb Sounder: Experiment overview, recovery, and validation of initial temperature data, J. Geophys. Res.-Atmos., 113, D16S43, doi:10.1029/2007JD008824, 2008.
Gong, J., Wu, D. L., and Eckermann, S. D.: Gravity wave variances and propagation derived from AIRS radiances, Atmos. Chem. Phys., 12, 1701-1720, doi:10.5194/acp-12-1701-2012, 2012.

Gordley, L. L., Marshall, B. T., and Chu, D. A.: Linepak: Algorithms for modeling spectral transmittance and radiance, J. Quant. Spectrosc. Ra., 52, 563-580, 1994.

Jiang, J. H., Eckermann, S., Wu, D., and Ma, J.: A search for mountain waves in MLS stratospheric limb radiances from the winter Northern Hemisphere: Data analysis and global mountain wave modeling, J. Geophys. Res.-Atmos., 109, D03107, doi:10.1029/2003JD003974, 2004.

Jiang, J. H., Eckermann, S. D., Wu, D. L., and Wang, D. Y.: Interannual variation of gravity waves in the Arctic and Antarctic winter middle atmosphere, Adv. Space Res., 38, 2418-2423, doi:10.1016/j.asr.2005.09.036, 2006.

Kalisch, S., Preusse, P., Ern, M., Eckermann, S. D., and Riese, M.: Differences in gravity wave drag between realistic oblique and assumed vertical propagation, J. Geophys. Res.-Atmos., 119, 10081-10099, doi:10.1002/2014JD021779, 2014.

Kim, J.-E. and Alexander, M. J.: Tropical Precipitation Variability and Convectively Coupled Equatorial Waves on Submonthly Time Scales in Reanalyses and TRMM, J. Climate, 26, 30133030, doi:10.1175/JCLI-D-12-00353.1, 2013.

Kim, Y.-J., Eckermann, S. D., and Chun, H.-Y.: An overview of the past, present and future of gravity-wave drag parameterization for numerical climate and weather prediction models - Survey article, Atmos.-Ocean, 41, 65-98, 2003.

Lane, T. P., Reeder, M. J., and Clark, T. L.: Numerical modeling of gravity wave generation by deep tropical convection, J. Atmos. Sci., 58, 1249-1274, 2001.

Lange, M. and Jacobi, C.: Analysis of gravity waves from radio occultation measurements, Springer, Berlin, 479-484, 2003.

Li, F., Austin, J., and Wilson, J.: The strength of the Brewer-Dobson circulation in a changing climate: Coupled chemistry-climate model simulations, J. Climate, 21, 40-57, doi:10.1175/2007JCLI1663.1, 2008.

Lindzen, R. S.: Turbulence and Stress Owing to Gravity Wave and Tidal Breakdown, J. Geophys. Res., 86, 9707-9714, 1981.

Marks, C. J. and Eckermann, S. D.: A three-dimensional nonhydrostatic ray-tracing model for gravity waves: formulation and preliminary results for the middle atmosphere, J. Atmos. Sci., 52, 1959-1984, 1995.

Marshall, B. T., Gordley, L. L., and Chu, D. A.: Bandpak: Algorithms for modeling broadband transmissions and radiance, J. Quant. Spectrosc. Ra., 52, 581-599, 1994.

Matsuno, T.: A quasi one-dimensional model of the middle atmosphere circulation interacting with internal gravity waves, J. Meteorol. Soc. Jpn., 60, 215-226, 1982.

McIntyre, M. E.: Breaking waves and global scale chemical transport in the Earth's atmosphere, with spinoffs for the Sun's interior, Prog. Theor. Phys., 130, 137-166, 1998.

McLandress, C.: On the importance of gravity waves in the middle atmosphere and their parameterization in general circulation models, J. Atmos. Terr. Phys., 60, 1357-1383, 1998.

McLandress, C. and Shepherd, T. G.: Simulated anthropogenic changes in the Brewer-Dobson circulation, including its extension to high latitudes, J. Climate, 22, 1516-1540, doi:10.1175/2008JCLI2679.1, 2009. 
McLandress, C., Alexander, M. J., and Wu, D. L.: Microwave Limb Sounder observations of gravity waves in the stratosphere: A climatology and interpretation, J. Geophys. Res., 105, 1194711967, 2000.

Mlynczak, M. G.: Energetics of the mesosphere and lower thermosphere and the SABER experiment, Adv. Space Res., 20, 11771183, 1997.

Orr, A., Bechtold, P., Scinocca, J., Ern, M., and Janiskova, M.: Improved middle atmosphere climate and forecasts in the ECMWF model through a nonorographic gravity wave drag parameterization, J. Climate, 23, 5905-5926, 2010.

Piani, C., Durran, D., Alexander, M. J., and Holton, J. R.: A Numerical Study of Three-Dimensional Gravity Waves Triggered by Deep Tropical Convection and Their Role in the Dynamics of the QBO, J. Atmos. Sci., 57, 3689-3702, 2000.

Preusse, P. and Ern, M.: Indication of convectively generated gravity waves observed by CLAES, Adv. Space Res., 35, 1987-1991, doi:10.1016/j.asr.2004.09.005, 2005.

Preusse, P., Eckermann, S. D., and Offermann, D.: Comparison of global distributions of zonal-mean gravity wave variance inferred from different satellite measurements, Geophys. Res. Lett., 27, 3877-3880, 2000.

Preusse, P., Eckermann, S., Oberheide, J., Hagan, M., and Offermann, D.: Modulation of gravity waves by tides as seen in CRISTA temperatures, in: Middle atmosphere temporal and spatial structures, edited by: Riese, M., Spang, R., Swenson, G. R., and Espy, P. J., vol. 27 of Advances in space research, 17731778, Deutsch Zentrum Luft Raumfahrt EU, European Space Agcy; Natl Aeronaut \& Space Adm, Stratospher Process \& Their Role Climate, Comm Space Res., Elsevier Science BV, Amsterdam, the Netherlands, doi:10.1016/S0273-1177(01)00336-2, C2 3/A1 4 Symposium of COSPAR Scientific Commission C held at 33rd COSPAR Scientific Assembly, 16-23 July 2000, Warsaw, Poland, 2001.

Preusse, P., Dörnbrack, A., Eckermann, S. D., Riese, M., Schaeler, B., Bacmeister, J. T., Broutman, D., and Grossmann, K. U.: Space-based measurements of stratospheric mountain waves by CRISTA, 1. Sensitivity, analysis method, and a case study, J. Geophys. Res., 107, 8178, doi:10.1029/2001JD000699, 2002.

Preusse, P., Ern, M., Eckermann, S. D., Warner, C. D., Picard, R. H., Knieling, P., Krebsbach, M., Russel III, J. M., Mlynczak, M. G., Mertens, C. J., and Riese, M.: Tropopause to mesopause gravity waves in August: measurement and modeling, J. Atmos. SolarTerr. Phys., 68, 1730-1751, 2006.

Preusse, P., Eckermann, S. D., and Ern, M.: Transparency of the atmosphere to short horizontal wavelength gravity waves, J. Geophys. Res., 113, D24104, doi:10.1029/2007JD009682, 2008.

Preusse, P., Eckermann, S. D., Ern, M., Oberheide, J., Picard, R. H., Roble, R. G., Riese, M., Russell III, J. M., and Mlynczak, M. G.: Global ray tracing simulations of the SABER gravity wave climatology, J. Geophys. Res., 114, D08126, doi:10.1029/2008JD011214, 2009a.

Preusse, P., Schroeder, S., Hoffmann, L., Ern, M., Friedl-Vallon, F., Ungermann, J., Oelhaf, H., Fischer, H., and Riese, M.: New perspectives on gravity wave remote sensing by spaceborne infrared limb imaging, Atmos. Meas. Tech., 2, 299-311, doi:10.5194/amt-2-299-2009, 2009b.

Preusse, P., Ern, M., Bechtold, P., Eckermann, S. D., Kalisch, S., Trinh, Q. T., and Riese, M.: Characteristics of gravity waves resolved by ECMWF, Atmos. Chem. Phys., 14, 10483-10508, doi:10.5194/acp-14-10483-2014, 2014.

Pulido, M. and Thuburn, J.: Gravity-wave drag estimation from global analyses using variational data assimilation principles, II: Case study, Q. J. Roy. Meteorol. Soc., 132, 1527-1543, 2006.

Remsberg, E. E., Marshall, B. T., Garcia-Comas, M., Krueger, D., Lingenfelser, G. S., Martin-Torres, J., Mlynczak, M. G., Russell III, J. M., Smith, A. K., Zhao, Y., Brown, C., Gordley, L. L., Lopez-Gonzalez, M. J., Lopez-Puertas, M., She, C. Y., Taylor, M. J., and Thompson, R. E.: Assessment of the quality of the Version 1.07 temperature-versus-pressure profiles of the middle atmosphere from TIMED/SABER, J. Geophys. Res., 113, D17101, doi:10.1029/2008JD010013, 2008.

Rienecker, M. M., Suarez, M. J., Gelaro, R., Todling, R., Bacmeister, J., Liu, E., Bosilovich, M. G., Schubert, S. D., Takacs, L., Kim, G.-K., Bloom, S., Chen, J., Collins, D., Conaty, A., da Silva, A., Gu, W., Joiner, J., Koster, R. D., Lucchesi, R., Molod, A., Owens, T., Pawson, S., Pegion, P., Redder, C. R., Reichle, R., Robertson, F. R., Ruddick, A. G., Sienkiewicz, M., and Woollen, J.: MERRA: NASA's Modern-Era Retrospective Analysis for Research and Applications, J. Climate, 24, 3624-3648, doi:10.1175/JCLI-D-11-00015.1, 2011.

Riese, M., Spang, R., Preusse, P., Ern, M., Jarisch, M., Offermann, D., and Grossmann, K. U.: CRISTA data processing and atmospheric temperature and trace gas retrieval, J. Geophys. Res., 104, 349-367, 1999.

Riese, M., Friedl-Vallon, F., Spang, R., Preusse, P., Schiller, C., Hoffmann, L., Konopka, P., Oelhaf, H., von Clarmann, T., and Höpfner, M.: GLObal limb Radiance Imager for the Atmosphere (GLORIA): Scientific objectives, Adv. Space Res., 36, 989-995, 2005.

Russell III, J. M., Mlynczak, M. G., Gordley, L. L., Tansock, J., and Esplin, R.: An overview of the SABER experiment and preliminary calibration results, Proc. SPIE, 3756, 277-288, 1999.

Salby, M. L.: Sampling theory for asynoptic satellite observatioons, Part I: Space-time spectra, resolution, and aliasing, J. Atmos. Sci., 39, 2577-2600, 1982.

Sigmond, M. and Scinocca, J. F.: The Influence of the Basic State on the Northern Hemisphere Circulation Response to Climate Change, J. Climate, 23, 1434-1446, doi:10.1175/2009JCLI3167.1, 2010.

Song, I. S. and Chun, H. Y.: Momentum flux spectrum of convectively forced internal gravity waves and its application to gravity wave drag parameterization, Part I: theory, J. Atmos. Sci., 62, 107-124, 2005.

Song, I.-S. and Chun, H.-Y.: A Lagrangian spectral parameterization of gravity wave drag induced by cumulus convection, J. Atmos. Sci., 65, 1204-1224, 2008.

Ungermann, J., Hoffmann, L., Preusse, P., Kaufmann, M., and Riese, M.: Tomographic retrieval approach for mesoscale gravity wave observations by the PREMIER Infrared Limb-Sounder, Atmos. Meas. Tech., 3, 339-354, doi:10.5194/amt-3-339-2010, 2010.

Wang, L. and Alexander, M. J.: Global estimates of gravity wave parameters from GPS radio occultation temperature data, J. Geophys. Res.-Atmos., 115, D21122, doi:10.1029/2010JD013860, 2010. 
Warner, D. C. and McIntyre, M. E.: An ultra-simple spectral parameterization for non-orographic gravity waves, J. Atmos. Sci., 58, 1837-1857, 2001.

Wright, C. J. and Gille, J. C.: Detecting overlapping gravity waves using the S-Transform, Geophys. Res. Lett., 40, 1850-1855, doi:10.1002/grl.50378, 2013.

Wright, C. J., Osprey, S. M., Barnett, J. J., Gray, L. J., and Gille, J. C.: High Resolution Dynamics Limb Sounder measurements of gravity wave activity in the 2006 Arctic stratosphere, J. Geophys. Res., 115, D02105, doi:10.1029/2009JD011858, 2010.

Wright, J. S. and Fueglistaler, S.: Large differences in reanalyses of diabatic heating in the tropical upper troposphere and lower stratosphere, Atmos. Chem. Phys., 13, 9565-9576, doi:10.5194/acp-13-9565-2013, 2013.
Wu, D. L. and Eckermann, S. D.: Global Gravity Wave Variances from Aura MLS: Characteristics and Interpretation, J. Atmos. Sci., 65, 3695-3718, doi:10.1175/2008JAS2489.1, 2008.

Wu, D. L. and Waters, J. W.: Gravity-wave-scale temperature fluctuations seen by the UARS MLS, Geophys. Res. Lett., 23, 32893292, 1996a.

Wu, D. L. and Waters, J. W.: Satellite observations of atmospheric variances: A possible indication of gravity waves, Geophys. Res. Lett., 23, 3631-3634, 1996b.

Wu, D. L. and Waters, J. W.: Observations of gravity waves with the UARS Microwave Limb Sounder, vol. 50, Springer, Berlin, Heidelberg, 103-120, 1997.

Wu, D. L., Preusse, P., Eckermann, S. D., Jiang, J. H., de la Torre Juarez, M., Coy, L., Lawrence, B., and Wang, D. Y.: Remote sounding of atmospheric gravity waves with satellite limb and nadir techniques, Adv. Space Res., 37, 2269-2277, 2006. 